\title{
Mechanisms of Hemispherically Symmetric Climate Variability*
}

\author{
Richard Seager, Nili Harnik, And Yochanan Kushnir \\ Lamont-Doherty Earth Observatory, Columbia University, Palisades, New York \\ WALTER ROBINSON \\ Department of Atmospheric Sciences, University of Illinois at Urbana-Champaign, Urbana, Illinois \\ JENNIFER MILLER \\ Lamont-Doherty Earth Observatory, Columbia University, Palisades, New York
}

(Manuscript received 7 August 2002, in final form 4 April 2003)

\begin{abstract}
Inspired by paleoclimate evidence that much past climate change has been symmetric about the equator, the causes of hemispherically symmetric variability in the recent observational record are examined using the National Centers for Environmental Prediction-National Center for Atmospheric Research reanalysis dataset and numerical models. It was found that the dominant cause of hemispherically symmetric variability is the El Niño-Southern Oscillation. During an El Niño event the Tropics warm at all longitudes and the subtropical jets in both hemispheres strengthen on their equatorward flanks. Poleward of the tropical warming there are latitude belts of marked cooling, extending from the surface to the tropopause in both hemispheres, at all longitudes and in all seasons. The midlatitude cooling is caused by changes in the eddy-driven mean meridional circulation. Changes in the transient eddy momentum fluxes during an El Niño event force upper-tropospheric ascent in midlatitudes through a balance between the eddy fluxes and the Coriolis torque. The eddy-driven ascent causes anomalous adiabatic cooling, which is primarily balanced by anomalous diabatic heating.

Using a quasigeostrophic spherical model, forced by an imposed surface eddy disturbance of chosen wavenumber and frequency, it is shown that the anomalous eddy momentum fluxes are caused by the impact that the changes in the tropically forced subtropical jets have on the propagation in the latitude-height plane of transient eddies. Changes in zonal winds, and associated changes in the meridional gradient of potential vorticity, create an anomalous region of low meridional wavenumber in the midlatitudes that refracts waves away both poleward and equatorward.

Tropical forcing of variability in the eddy-driven mean meridional circulation is another way, in addition to Rossby wave teleconnections, whereby the Tropics can influence extratropical climate. Unlike teleconnections this mechanism causes climate variability that has strong zonally and hemispherically symmetric components and operates throughout the seasonal cycle.
\end{abstract}

\section{Introduction}

There is increasing evidence that important components of the earth's climate variability, on timescales from the interannual to the glacial, occur symmetrically about the equator. The case of the El Niño-Southern Oscillation (ENSO) is the most familiar. Garreaud and Battisti (1999) show the impressive degree of symmetry about the equator of the anomalies of sea surface temperature (SST), surface winds, and sea level pressure that occur on interannual timescales associated with

* Lamont-Doherty Earth Observatory Contribution Number 6463.

Corresponding author address: Dr. Richard Seager, Lamont-Doherty Earth Observatory, Columbia University, Palisades, NY 10964. E-mail: rich@maatkure.ldeo.columbia.edu
ENSO. The symmetry arises in the forcing of the circulation anomalies in the equatorial region and the subsequent forcing of SST anomalies in the extratropics by surface fluxes (e.g., Lau and Nath 1996). Within the Tropics themselves ENSO gives rise to spatially uniform changes of tropospheric temperature that are also symmetric about the equator (Yulaeva and Wallace 1994). Garreaud and Battisti (1999) show that the decadal ENSO-like variability is basically symmetric about the equator with the interesting exception of strong SST anomalies in the North Pacific along the latitude of the Kuroshio-Oyashio Extension. The latter feature has been explained by the adjustment of the wind-driven ocean gyre circulation, which is quite different between the North Pacific and South Pacific (Schneider et al. 2002; Seager et al. 2001).

On timescales of hundreds of years to millennia there 
is also evidence that climate variations occurred symmetrically about the equator. During the Little Ice Age the glacial advance in New Zealand was as extensive as that in Europe (Wardle 1973; Winkler 2000) and there were obvious glacial advances throughout the Tropics (Kaser 1999). The dramatic cooling during the Younger Dryas period, which interrupted the most recent deglaciation is as clearly present in New Zealand as in Europe (Denton and Hendy 1994). Furthermore, details of the progress of the last deglaciation correspond closely in time between southern Chile and the European Alps despite opposite phased changes in local insolation (Lowell et al. 1995). Markgraf et al. (2000) discuss evidence from lake records that symmetric climate changes in North and South America occurred in the late glacial and Holocene that they suggest were linked to changes in the latitude of the westerlies.

Hemispherically symmetric climate variability will almost certainly involve an active role for the Tropics. In the recent climate record of the last $50 \mathrm{yr}$ we will show that essentially all hemispherically symmetric variations are forced by ENSO. Over longer periods of time, other phenomena that actively impact the Tropics, such as variations in the earth's orbit (Clement et al. 1999, 2001), the trace gas content of the atmosphere, or near-global reorganizations of the ocean circulation (Bush and Philander 1998) could trigger hemispherically symmetric variations.

In the current work we will use the National Centers for Environmental Prediction-National Center for Atmospheric Research (NCEP-NCAR) reanalysis (Kalnay et al. 1996; Kistler et al. 2001) to examine the mechanisms whereby hemispherically symmetric variability occurs. In practice this will mean examining how tropical forcing by ENSO sets in motion a sequence of events causing changes in atmospheric circulation and temperatures that are, to first order, the same in both hemispheres. A major component of this work will be to explain why during an El Niño event the Tropics warm but the extratropics cool (except in Northern Hemisphere during local winter) and the opposite occurs during a La Niña event. The opposite-signed extratropical response to ENSO has previously been noticed by Robinson (2002), is also seen in Yulaeva and Wallace (1994) and is discussed by Hoerling et al. (2001). It will be shown that changes in the Hadley cell strength related to ENSO cause an acceleration (El Niño) or deceleration (La Niña) of the subtropical jets, as previously noted by Arkin (1982). Transient eddies then propagate in an altered mean zonal flow and the resulting changes in eddy momentum fluxes cause bands of extratropical eddy-driven ascent and descent that force changes in tropospheric temperatures. These mechanisms are likely to be relevant for hemispherically symmetric climate variations forced by other means. Chang (1995) has previously demonstrated, within the context of an idealized model, how changes in the Hadley cell intensity can affect midlatitude climate via the impact on transient eddies and the eddy-driven mean meridional circulation.

The plan of the paper is as follows. In section 2 we form an index of hemispherically symmetric circulation variability by analyzing the zonal-mean zonal wind at $300 \mathrm{mb}$. The index is highly correlated to ENSO and describes a hemispherically symmetric equatorward and poleward movement of the subtropical jets in both hemispheres. In association with this are bands in the extratropics where the temperature change is of opposite sign to that in the Tropics. We then examine the terms in the momentum and temperature budgets to see what processes maintain these variations. In section 3 we demonstrate that the same variability is captured in an ensemble of atmosphere GCM integrations subjected to the observed history of SSTs as a lower boundary condition, proving that this is a boundary-forced phenomena. In section 4 we perform a simple calculation to demonstrate that the extratropical temperature signal arises within an eddy-induced mean-meridional circulation. In section 5 we alter the basic-state zonal winds in a simple quasigeostrophic model and demonstrate how this impacts the propagation in the latitude-height plane of transient eddies resulting in changes in eddy fluxes, the mean meridional circulation, and the temperature that are similar to those observed. Discussion of the relationship to prior results is offered in section 6 and the conclusions are given in section 7 .

\section{Observational analyses}

All of the observational analyses are based on data from the NCEP-NCAR reanalyses for the period from 1979 to 2001. We use only this recent period because of the inclusion of satellite data in the reanalyses, greatly improving data coverage in the Southern Hemisphere. All computations have also been done for the complete period since 1949 . The results were essentially the same in temporal and spatial behavior as for the recent period. However, in the recent period of the reanalyses the climate variations appear larger and more statistically significant.

\section{a. Analysis of variability in tropospheric zonal winds and temperature}

Hemispherically symmetric variability of the atmospheric circulation, transient eddy behavior and temperatures will be closely tied together. A fundamental element will be variability in the strength and latitude of the subtropical jet streams that form on the poleward flank of the Tropics in both hemispheres. We begin with an analysis of the zonal mean zonal wind at $300 \mathrm{mb}$, which is close to being the pressure level of strongest zonal winds. To retain the seasonal evolution of the variability we perform an EOF analysis in the latitudecalendar month domain. This analysis returns a series of EOFs describing the structure in latitude and calendar 
(a) Regression to mean zonal wind $300 \mathrm{mb}$

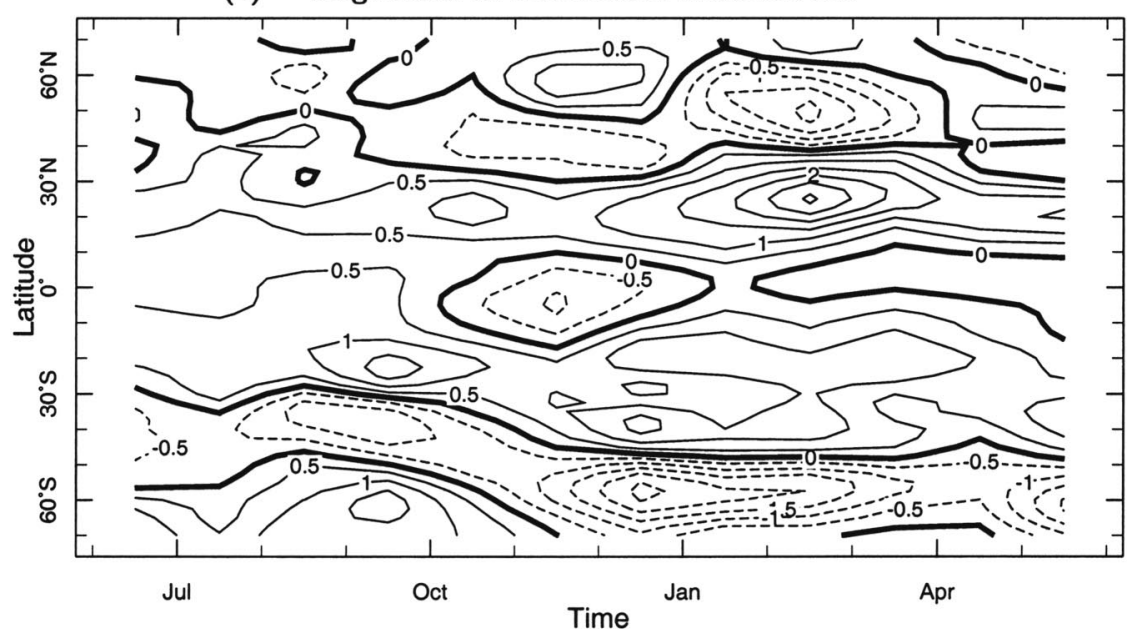

(b) U 300 mb PC 1 Jun-May

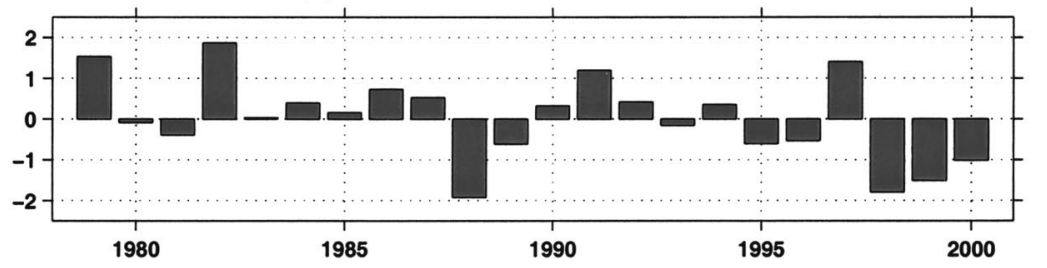

FIG. 1. (a) The first EOF of the 300-mb zonal mean zonal wind $\left(\mathrm{m} \mathrm{s}^{-1}\right)$ from an analysis performed in the latitude-calendar month domain. (b) The corresponding PC.

month and corresponding principal components that have one value per year. ${ }^{1}$ The first EOF and its principal component is shown in Fig. 1. The principal component (PC, hereafter referred to as the wind index) correlates with the Niño-3 SST index (the spatially averaged SST anomaly between $5^{\circ} \mathrm{S}$ and $5^{\circ} \mathrm{N}$ and $150^{\circ}$ and $90^{\circ} \mathrm{W}$ ) at 0.79 indicating that a positive wind index corresponds to El Niño conditions. The first EOF explains $13.5 \%$ of the variance of the zonal mean $300-\mathrm{mb}$ zonal wind.

The latitude structure of the EOF clearly describes bands that are nearly symmetric about the equator. During an El Niño zonal mean zonal winds at $300 \mathrm{mb}$ strengthen between about $20^{\circ}$ and $40^{\circ}$, and weaken between about $40^{\circ}$ and $60^{\circ}$, north and south of the equator. These variations in the atmospheric circulation are closely associated with variations in the temperature. Figure 2 shows the zonal mean thickness temperature between 300 and $850 \mathrm{mb}$ regressed onto the wind index. During an El Niño the Tropics warm as expected given the increase in tropical Pacific SST and the anomalous heat flux from ocean to atmosphere (Sun 2000). Harder to explain is the extensive cooling that occurs in the extratropics (Robinson 2002; Hoerling et al. 2001). The extratropical cooling is most persistent and apparent in the $30^{\circ}$ to $60^{\circ}$ latitude band in both hemispheres.

\footnotetext{
${ }^{1}$ The year is centered on Northern Hemisphere winter, so, for example, the year labeled 1980 runs from June 1980 to May 1981.
}

The zonally symmetric signal in zonal winds and temperature is not an artifact of averaging over a longitudinally varying pattern. In Figs. 3a-d we show the global patterns of zonal wind at $300 \mathrm{mb}$ (contours) and thickness temperature (colors) between 300 and $850 \mathrm{mb}$ regressed onto the wind index for the seasons of JuneJuly-August (JJA), September-October-November (SON), December-January-February (DJF) and March-April-May (MAM). While the wind anomalies maximize in the Pacific sector there is also a noticeable zonally symmetric component to the wind variability in both hemispheres, and in all seasons. Zonal symmetry is particularly marked in the Southern Hemisphere. The tropical warming is centered over the Pacific but extends to all longitudes and is most marked in DJF and MAM, when ENSO events tend to peak. The extratropical cooling during El Niño is a remarkably robust signal and has an obvious zonally symmetric component.

The vertical structure of these variations is shown in Figs. 4a-d where the zonal means of the zonal wind and temperature, regressed onto the wind index, are shown for the four seasons. The extratropical areas of strongest temperature and wind changes have a correlation coefficient with the wind index of 0.6 or more that is significant at the $99 \%$ level, confirming the robustness of the signal. The temperature variations are vertically coherent in the Tropics and extratropics. Both temperature and wind variations, and the degree of 


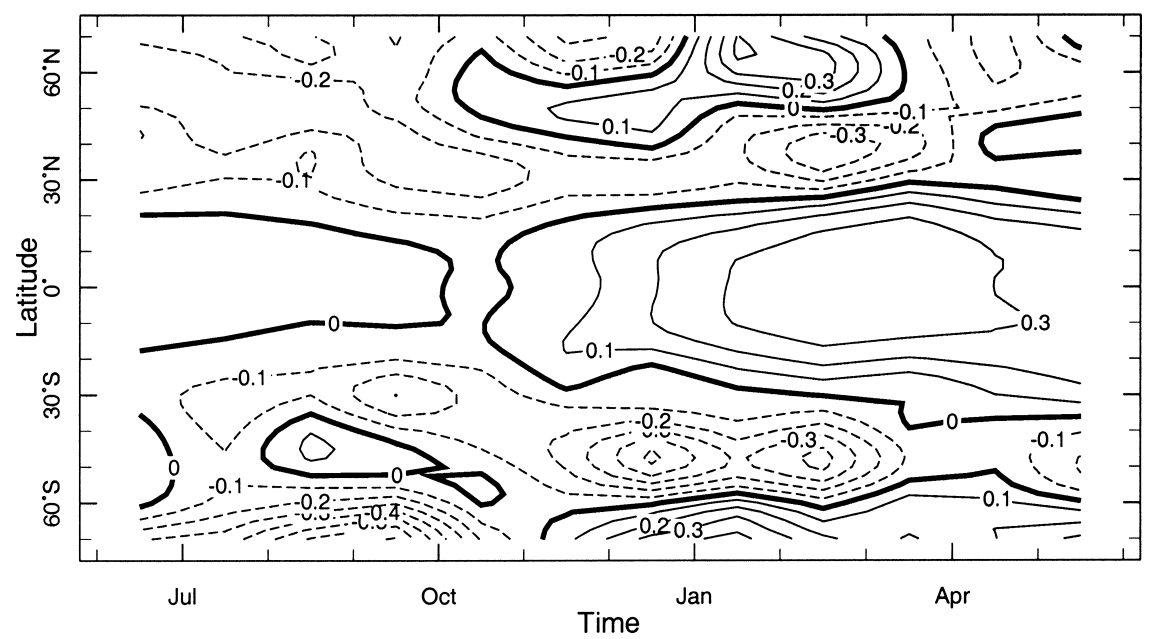

FIG. 2. The zonal mean thickness temperature (K) between 300 and $850 \mathrm{mb}$ regressed onto the time series of Fig. $1 \mathrm{~b}$.

hemispheric symmetry, are weak in the Southern Hemisphere during MAM and JJA because of only weak zonal symmetry (Figs. 3a,d) at this time. The variations in the zonal-mean zonal wind are also vertically coherent, peaking in the upper troposphere but also influencing the surface winds. The zonal wind anomalies relate to the temperature anomalies by thermal wind balance: anomalous westerlies occur where the meridional temperature gradient strengthens and anomalous easterlies where the temperature gradient weakens. ${ }^{2}$ Strengthening of the westerlies on the poleward flank of the El Niño-induced tropical warming is no surprise. Explaining the weaker westerlies at higher latitudes will require an explanation for why the midlatitudes cool.

\section{b. Analysis of variability in the tropospheric zonal momentum and heat budgets}

The next task is to understand how the variations in the zonal-mean zonal winds and temperatures occur. Using the NCEP-NCAR reanalysis we compute the terms in the zonal-mean zonal wind and temperature equations, dividing them into contributions from the zonalmean circulation, stationary waves, and transient eddies. The equation for the zonal-mean zonal wind is

$$
\begin{aligned}
\frac{\partial\langle\bar{u}\rangle}{\partial t}= & -\left(\frac{\langle\bar{v}\rangle}{a} \frac{\partial\langle\bar{u}\rangle}{\partial \phi}+\langle\bar{\omega}\rangle \frac{\partial\langle\bar{u}\rangle}{\partial p}\right)+\left(f+\frac{\langle\bar{u}\rangle \sin \phi}{a \cos \phi}\right)\langle\bar{v}\rangle \\
& -\frac{1}{a \cos ^{2} \phi} \frac{\partial}{\partial \phi}\left(\left\langle\bar{u}^{*} \bar{v}^{*}\right\rangle \cos ^{2} \phi\right)-\frac{\partial}{\partial p}\left\langle\bar{u}^{*} \bar{\omega}^{*}\right\rangle
\end{aligned}
$$

\footnotetext{
${ }^{2}$ Apparently the entire stratosphere warms during an El Niño event. This warming is reproduced in the SST-forced atmospheric general circulation model results described in section 3. While it is of considerable interest, any explanation is beyond the scope of this work.
}

$$
\begin{aligned}
& -\frac{1}{a \cos ^{2} \phi} \frac{\partial}{\partial \phi}\left(\left\langle\overline{u^{\prime} v^{\prime}}\right\rangle \cos ^{2} \phi\right)-\frac{\partial}{\partial p}\left\langle\overline{u^{\prime} \omega^{\prime}}\right\rangle \\
& -\overline{D\langle u\rangle} .
\end{aligned}
$$

Here, angle brackets indicate a zonal mean, the asterisk indicates departures from zonal mean, overbars indicate a monthly mean, and primes indicate departures from monthly means. The variable $u$ is the zonal wind, $v$ the meridional wind, and $\omega$ the vertical pressure velocity; $a$ is the radius of the earth, $\phi$ is latitude, $p$ is pressure, $f$ is the Coriolis parameter, and $\overline{D\langle u\rangle}$ is a damping. The first right-hand-side term on the first line of Eq. (1) is the advection of the zonal-mean wind by the mean-meridional circulation, the second term incorporates the Coriolis torque, the third and fourth terms are the forcing by momentum flux convergence within the stationary waves, and the fifth and sixth terms are the forcing by momentum flux convergence within the transient eddies.

The temperature equation is

$$
\begin{aligned}
\frac{\partial\langle\bar{T}\rangle}{\partial t}= & -\left[\frac{\langle\bar{v}\rangle}{a} \frac{\partial\langle\bar{T}\rangle}{\partial \phi}+\langle\bar{\omega}\rangle\left(\frac{\partial\langle\bar{T}\rangle}{\partial p}-\frac{R\langle\bar{T}\rangle}{c_{p} p}\right)\right] \\
& -\frac{1}{a \cos \phi} \frac{\partial}{\partial \phi}\left(\left\langle\bar{v}^{*} \bar{T}^{*}\right\rangle \cos \phi\right)-\frac{\partial}{\partial p}\left\langle\bar{\omega}^{*} \bar{T}^{*}\right\rangle \\
& +\frac{R\left\langle\bar{\omega}^{*} \bar{T}^{*}\right\rangle}{c_{p} p}-\frac{1}{a \cos \phi} \frac{\partial}{\partial \phi}\left(\left\langle\overline{\bar{v}^{\prime} T^{\prime}}\right\rangle \cos \phi\right) \\
& -\frac{\partial}{\partial p}\left\langle\overline{\omega^{\prime} T^{\prime}}\right\rangle+\frac{R\left\langle\overline{\omega^{\prime} T^{\prime}}\right\rangle}{c_{p} p}+\langle Q\rangle .
\end{aligned}
$$

Here, $T$ is temperature, $R$ is the gas constant, $c_{p}$ the specific heat at constant pressure, and $\langle Q\rangle$ is the diabatic heating, evaluated as a residual. As in Eq. (1) the terms are divided into contributions from the mean-meridional circulation, stationary waves, and transient eddies. 
(a) JJA

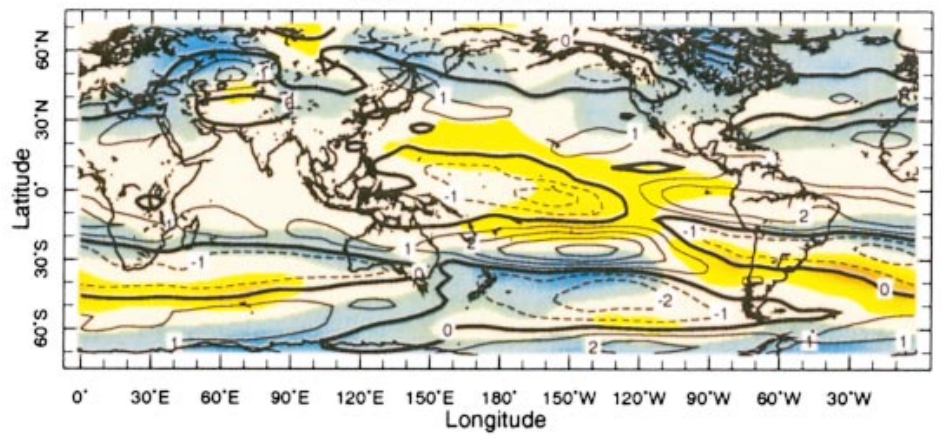

(b) SON

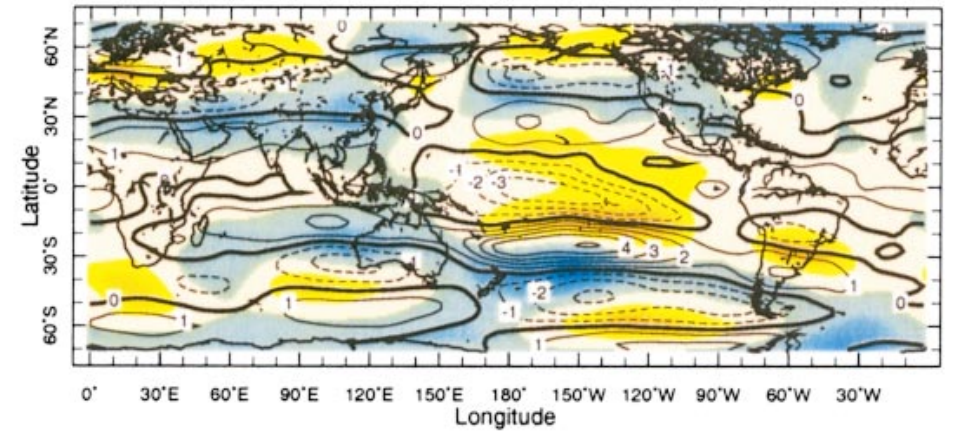

(c) DJF

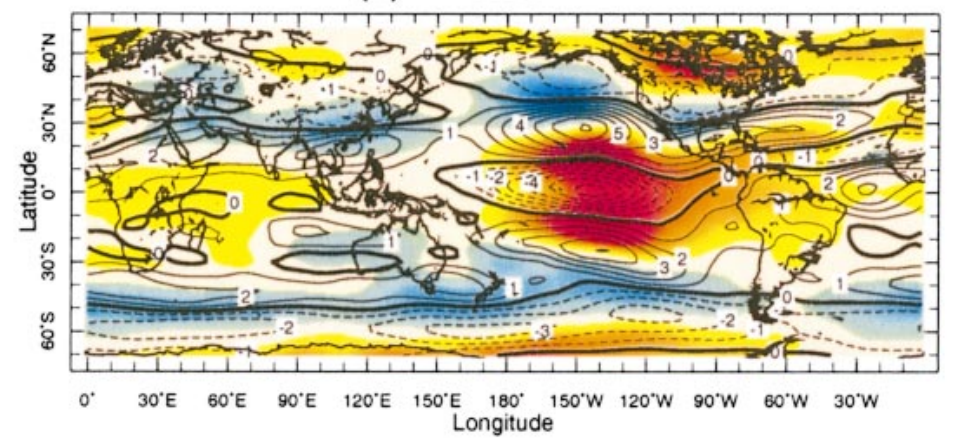

(d) MAM
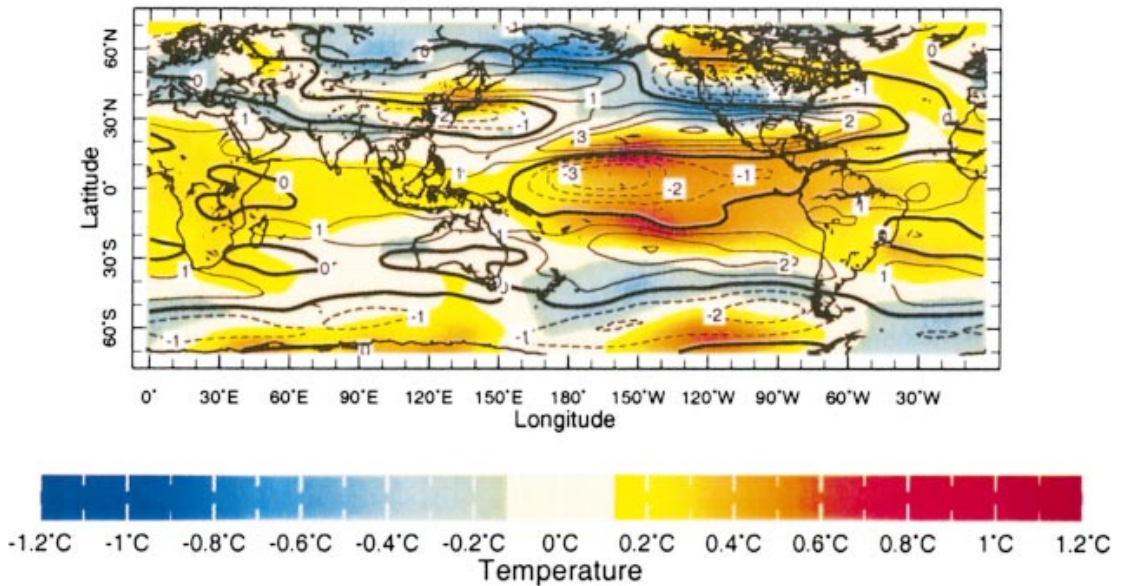
Each of the terms in Eqs. (1) and (2) was computed using data from the NCEP-NCAR reanalyses at all pressure levels for the 1979-2001 period. The variable $\langle Q\rangle$ is evaluated as a residual but $\overline{D\langle u\rangle}$ is not evaluated. Monthly anomalies were computed by subtracting the monthly climatology over this period of the relevant term. The monthly anomalies were then regressed onto the wind index.

In the interest of brevity we will only show results for SON and DJF, which both have noticeable hemispherically symmetric variability. The variability of mean advection of zonal momentum is only significant in the upper troposphere of the deep Tropics, where it is a significant component of the Hadley cell circulation, but is not shown here. The stationary eddy heat flux convergence also does not contribute to the hemispherically symmetric patterns under consideration and is not shown.

Figure 5 shows the terms in the zonal momentum equation related to the Coriolis effect, stationary waves, and transient eddies for SON. Figure 6 shows the same quantities for DJF. To the extent that the flow is inviscid the zonal mean zonal winds are, to first order, in geostrophic balance with the meridional geopotential height gradient. In this case we do not expect to see much correspondence between the variability of the zonal wind and the variability of the terms in the zonal momentum budget. This is the case in the subtropics where the strengthening of the jets during El Niño (weakening during La Niña) is clearly related to the strengthened pole to equator temperature gradient. In the extratropics, however, it is clear that transient eddy momentum flux convergence is helping force the changes in the zonal mean zonal wind. Of more interest is the near-ubiquitous close cancellation between the transient eddy momentum flux convergence and the Coriolis torque. For example in the Southern Hemisphere during SON in the upper troposphere the transient eddies are applying, a westerly force north of $30^{\circ} \mathrm{S}$ and an easterly force south of there. This is closely balanced by the Coriolis term implying northward flow north of $30^{\circ} \mathrm{N}$ and southward flow to the south, that is, upper-troposphere divergence. This same balance is weakly evident in the Northern Hemisphere during SON and very clear in both hemispheres during DJF, in all cases forcing upper-tropospheric divergence somewhere in the $30^{\circ}-60^{\circ}$ latitude belt. During DJF the stationary eddy momentum flux convergence (Fig. 6b) augments the role of the transients but is not important in other seasons or south of the equator.

Figures 7 and 8 show the terms in the temperature equation related to the mean advection, the diabatic heating (evaluated as the residual), and transient eddy heat flux convergence for SON and DJF. The primary balance in the Tropics and extratropics is between the mean advection and the diabatic heating. In the Tropics El Niño is associated with increased diabatic heating near the equator and increased mean advective cooling, indicating increased vertical ascent to balance the heating. In the subtropics there is increased mean advective warming (by subsidence) balancing increased diabatic cooling (presumably by radiation although reduced condensational heating could also be important). These are the expected ENSO relationships and indicate a strengthening of the thermally direct Hadley cell during El Niño.

In the extratropics the temperature budget is quite different. The bands of cool air during El Niño are being created by mean advective cooling and being damped by the diabatic heating. Bands of mean advective cooling match the regions of transient eddy-induced uppertropospheric divergence, and presumed ascent, as seen in Figs. 5 and 6. Consequently, temperature changes in the Tropics are being created by diabatic heating and this forces the changes in the atmospheric circulation. In the extratropics the mean-meridional atmospheric circulation is being forced by the eddy momentum fluxes and is creating temperature anomalies that the diabatic heating is attempting to damp. The transient eddy heat flux (Figs. 7c and 8c) is also attempting to damp the temperature perturbations.

\section{Simulation of ENSO-related hemispherically symmetric climate variability in a climate model}

Before explaining the observed patterns of variations in zonal mean temperature and atmospheric circulation, we note that they are well reproduced in a climate model forced by observed SSTs. A 24-member ensemble of SST-forced experiments was performed at the International Research Institute for Climate Prediction with the ECHAM-4.5 atmospheric GCM (Roeckner et al. 1996) for a period that includes the 1979-2001 period analyzed in the observations. The model has a triangular truncation at wavenumber 42, and 19 levels in the vertical. We performed the same analysis on the model results as we have presented for the observations, beginning with a calendar month and latitude EOF decomposition of the variations in zonal mean zonal winds at $300 \mathrm{mb}$. The EOF (not shown) displays the same hemispherically symmetric variations in the winds as seen in the observations. The model wind index, defined as the PC of the first EOF, has a correlation coefficient with the Niño-3 index, constructed from the SSTs used to force the model, of 0.84 . The EOF explains $48 \%$ of the variance of the zonal mean 300-mb zonal wind. This

$\leftarrow$

FIG. 3. The zonal wind (contours, $\mathrm{m} \mathrm{s}^{-1}$ ) and thickness temperature between 300 and 850 mb (colors, K) both regressed onto the time series of Fig. 1 b for (a) JJA, (b) SON, (c) DJF, and (d) MAM. 


\section{(a) JJA}

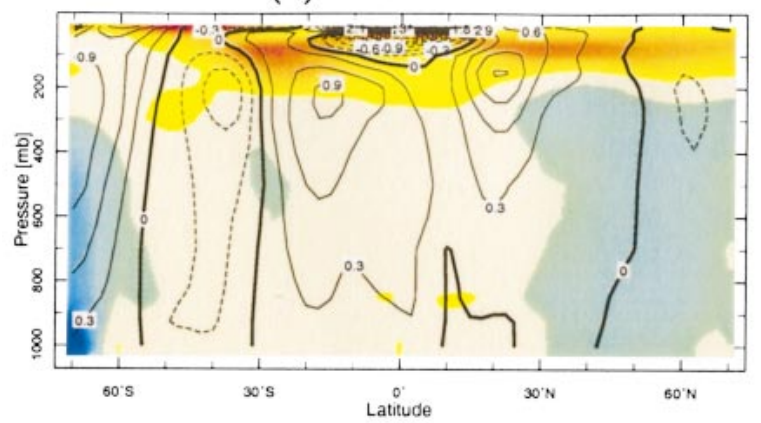

(b) SON

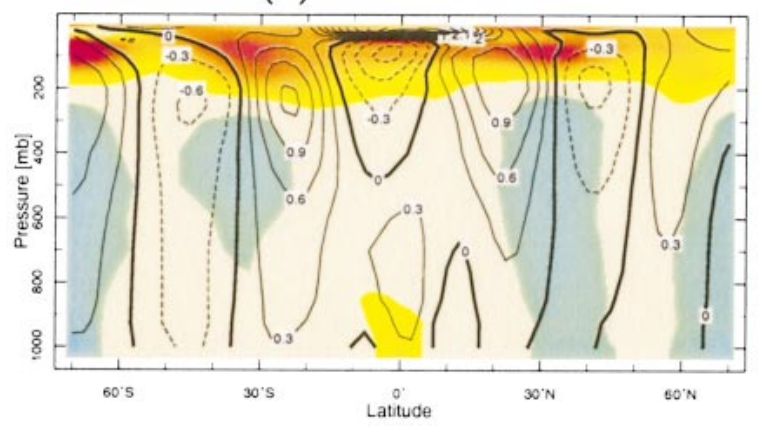

(c) DJF

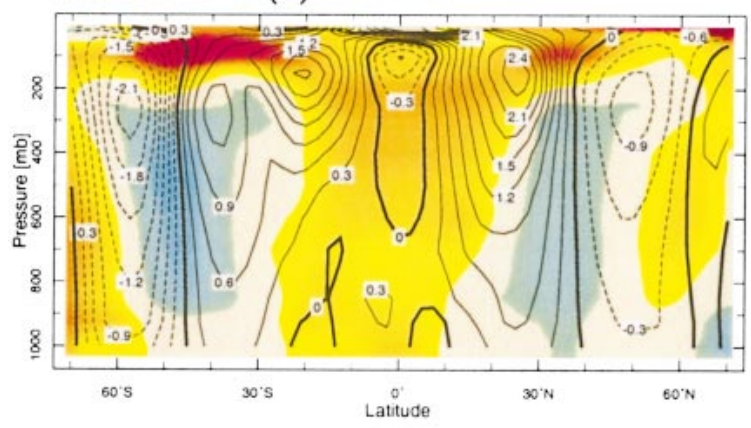

(d) MAM

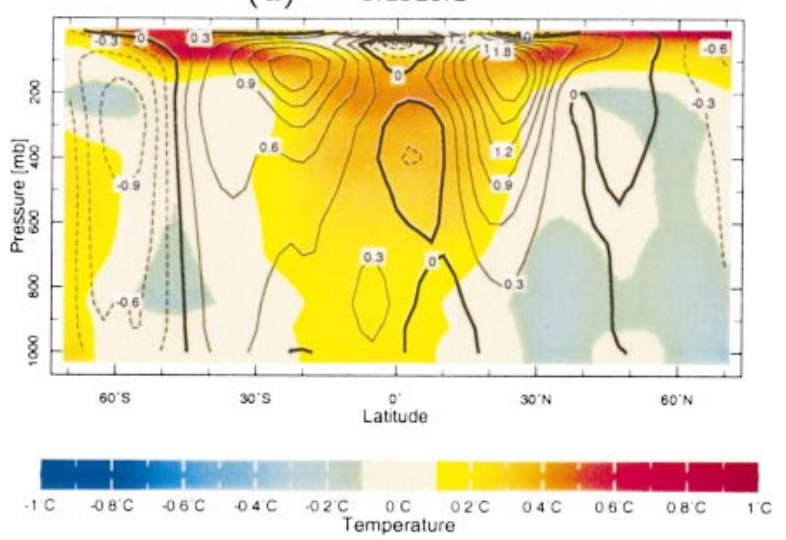

FIG. 4. The zonal-mean zonal wind (contours, $\mathrm{m} \mathrm{s}^{-1}$ ) and zonalmean temperature (colors, $\mathrm{K}$ ) both regressed onto the time series of Fig. $1 \mathrm{~b}$ for (a) JJA, (b) SON, (c) DJF, and (d) MAM. (a) Momentum Budget SON Coriolis

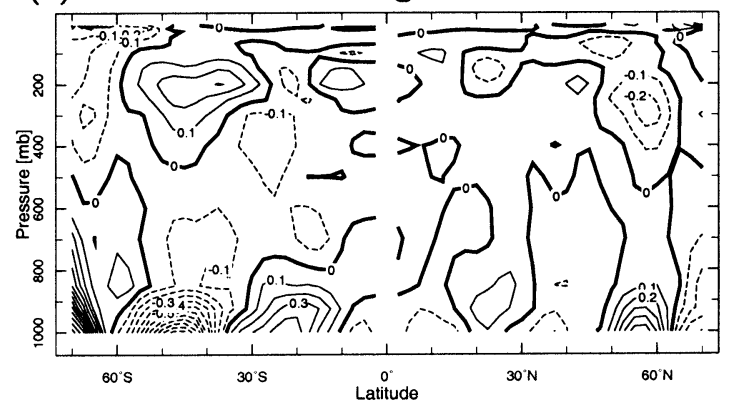

(b)

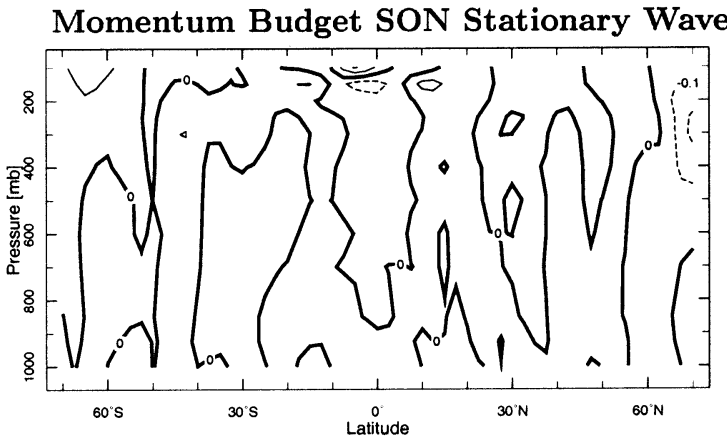

(c)

Momentum Budget SON Transient Eddies

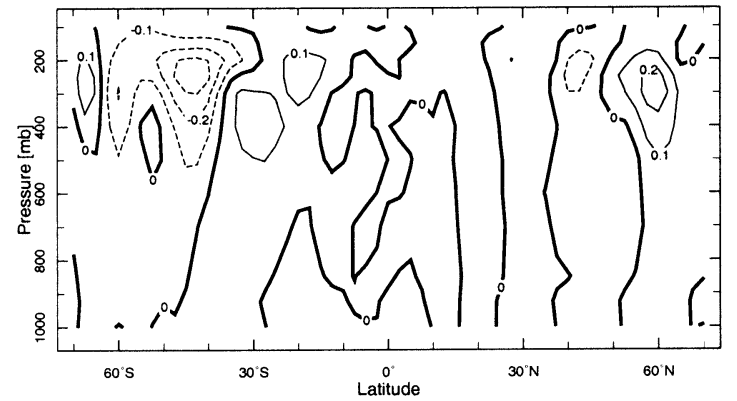

FIG. 5. Tendencies of the zonal mean zonal wind $\left(\mathrm{m} \mathrm{s}^{-1} \mathrm{day}^{-1}\right)$ due to (a) the Coriolis torque, (b) convergence of momentum by stationary waves, and (c) convergence of momentum by transient eddies for SON derived by regression of these quantities on the zonal wind index.

is a much larger amount than the first observed EOF explained because the ensemble average isolates the boundary-forced variability.

In Figure 9 we show the zonal-mean temperature and zonal wind regressed onto the model zonal wind index for the four seasons. The model Tropics warm during El Niño, apparently by more than is observed, and in both hemispheres the subtropical jets strengthen. The model also reproduces the bands of cooling in the midlatitudes of both hemispheres. The location, magnitude, and vertical structure of the coolings are similar to those observed.

Given the size of the ensemble ( 24 members) it is highly probable that the ensemble mean is the SSTforced response. This, in combination with the high cor- 
(a) Momentum Budget DJF Coriolis

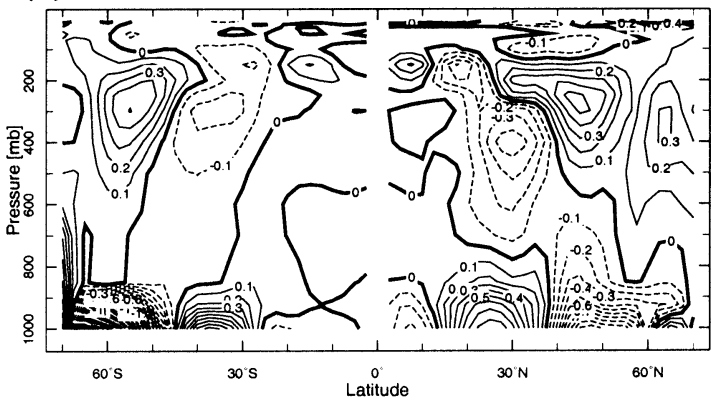

(b) Momentum Budget DJF Stationary Wave

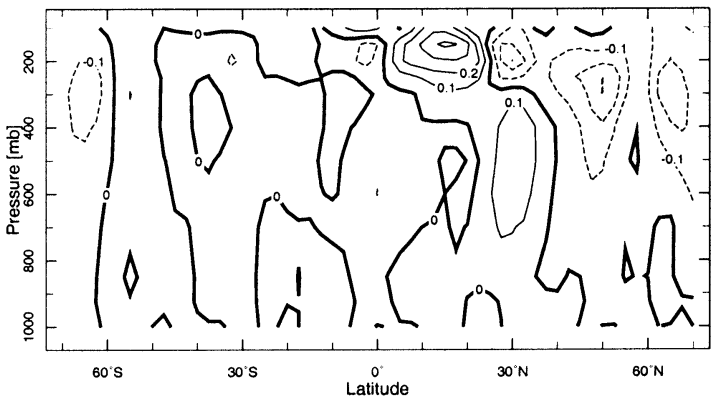

(c) Momentum Budget DJF Transient Eddies

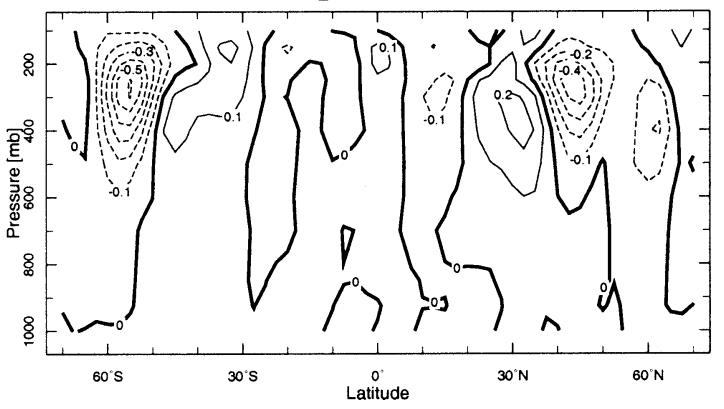

FIG. 6. Same as in Fig. 5 but for DJF.

relation between the zonal wind index and Niño-3 makes it clear that the hemispherically symmetric variability and the midlatitude cooling is a forced response to ENSO. $^{3}$

\section{Coupling between transient eddies and the mean-meridional circulation and the creation of extratropical cooling during EI Niño}

The analysis in the previous section suggests that transient eddy momentum fluxes are playing a critical

\footnotetext{
${ }^{3}$ We also performed two experiments with the NCAR Community Climate Model in which we applied El Niño and La Niña SST conditions permanently, that is, all the year round, in the tropical Pacific Ocean with climatological SSTs elsewhere. In those experiments the midlatitudes were cooler for permanent El Niño conditions than for permanent La Niña conditions, indicating that this is a steady response to tropical forcing and is not associated with slow propagation of signals from the Tropics into midlatitudes as suggested by Dickey et al. (1992).
}

(a)

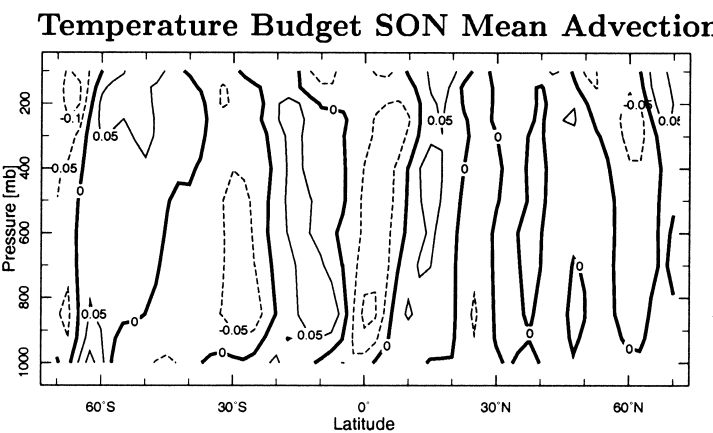

(b)
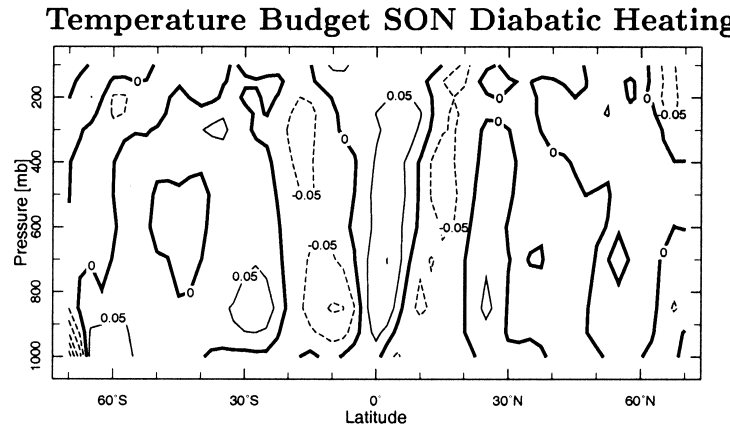

(c)

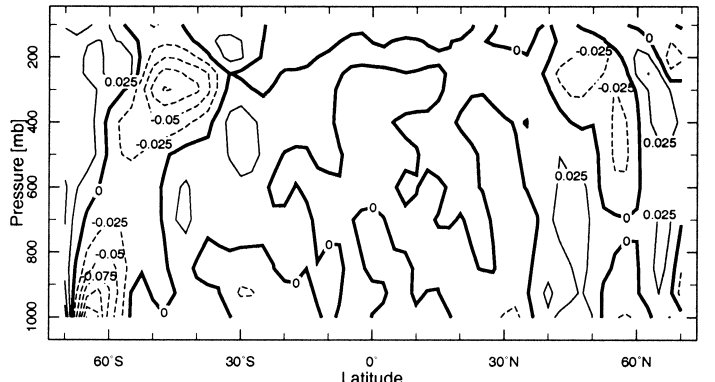

FIG. 7. Tendencies of the zonal mean temperature $\left(\mathrm{K} \mathrm{day}^{-1}\right)$ due to (a) advection by the zonal-mean circulation, (b) the diabatic heating (evaluated as a residual), and (c) transient eddy heat flux convergence for SON derived by regression of these quantities on the zonal wind index.

role in providing the link between changes in tropical and extratropical tropospheric temperatures. However this is not by their heat transport, which merely acts to weaken the anomalous pole-to-equator temperature gradient. Instead it is by their momentum transport and the impact that this has on the mean meridional circulation.

To leading order, the zonally averaged governing equations in the extratropics are

$$
\begin{aligned}
-\left(f+\frac{\langle\bar{u}\rangle \sin \phi}{a \cos \phi}\right)\langle\bar{v}\rangle & =-\frac{1}{a \cos ^{2} \phi} \frac{\partial}{\partial \phi}\left(\left\langle\overline{u^{\prime} v^{\prime}}\right\rangle \cos ^{2} \phi\right) \\
\left(f+\frac{\langle\bar{u}\rangle \sin \phi}{a \cos \phi}\right)\langle\bar{u}\rangle & =-\frac{1}{a} \frac{\partial\langle\bar{\Phi}\rangle}{\partial \phi}
\end{aligned}
$$


(a)

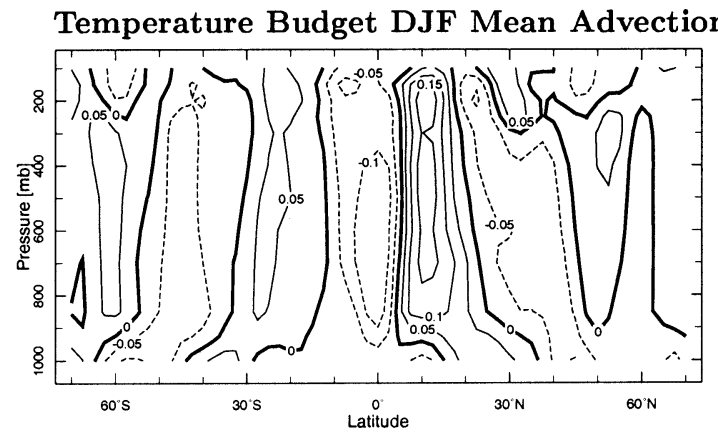

(b)

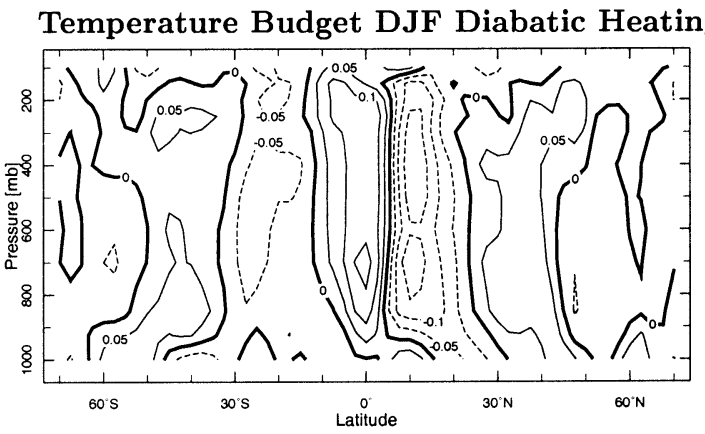

(c)

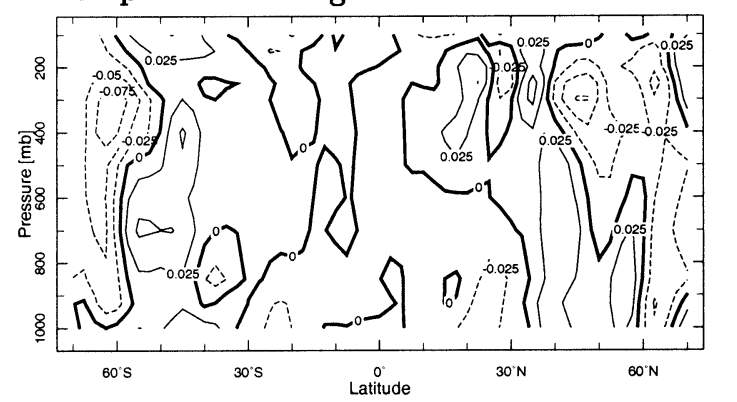

FIG. 8. Same as in Fig. 7 but for DJF.

$$
\begin{gathered}
\frac{1}{a \cos \phi} \frac{\partial\langle\bar{v}\rangle \cos \phi}{\partial \phi}+\frac{\partial\langle\bar{\omega}\rangle}{\partial p}=0 \\
\langle\bar{\omega}\rangle\left(\frac{\partial\langle\bar{T}\rangle}{\partial p}-\frac{R\langle\bar{T}\rangle}{c_{p} p}\right)=-\frac{1}{a \cos \phi} \frac{\partial}{\partial \phi}\left(\left\langle\overline{v^{\prime} T^{\prime}}\right\rangle \cos \phi\right) \\
-\frac{\partial}{\partial p}\left\langle\overline{\omega^{\prime} T^{\prime}}\right\rangle+\frac{R\left\langle\overline{\omega^{\prime} T^{\prime}}\right\rangle}{c_{p} p}-\alpha\langle\bar{T}\rangle .
\end{gathered}
$$

Here, $\Phi$ is the geopotential. The diabatic heating has been written as a Newtonian cooling with an inverse timescale $\alpha$ to emphasize that this is a damping term.

The sequence of events during an El Niño is then as follows. First, the tropical troposphere warms in response to warmer sea surface temperatures and anomalous surface heat flux from the ocean to the atmosphere. This increases the magnitude of $\partial\langle\bar{\Phi}\rangle / \partial \phi$, the pole to equator temperature gradient, and the zonal-mean zonal jet strengthens at around $20^{\circ}$ latitude in both hemi- spheres. This alters the basic state in which transient eddies propagate, altering the pattern of transient eddy momentum flux convergence. By the balance in Eq. (3) the altered momentum flux convergence sets up patterns of upper-tropospheric divergence and convergence. By continuity, Eq. (5), this divergence is balanced by ascent and convergence by descent. Eddy-induced ascent and descent force cooling and warming by the balance in Eq. (6), while the transient eddy heat transport attempts to smooth these out and the diabatic heating attempts to damp them.

The first way to check this line of causality is to compare the eddy-induced vertical motion with the observed motion. From Eqs. (3) and (5) the eddy-induced vertical motion is found to be

$$
\begin{aligned}
\langle\bar{\omega}(p)\rangle=\left\langle\overline{\omega_{0}}\right\rangle- & \frac{1}{a \cos \phi} \frac{\partial}{\partial \phi} \\
\times \int_{p_{0}}^{p} & \frac{1}{a\left(f+a^{-1}\langle\bar{u}\rangle \tan \phi\right)} \frac{\partial}{\partial \phi} \\
& \times\left(\left\langle\overline{u^{\prime} v^{\prime}}\right\rangle \cos ^{2} \phi\right) d p .
\end{aligned}
$$

Here, $\left\langle\bar{\omega}_{0}\right\rangle$ is the zonal-mean pressure velocity at an upper-troposphere level, taken to be $100 \mathrm{mb}$. The eddyinduced $\langle\bar{\omega}(p)\rangle$ was computed at all levels using $\overline{u^{\prime} v^{\prime}}$ from the reanalysis. In Fig. 10 we show the observed zonal mean vertical pressure velocity and zonal mean temperature for SON and DJF both regressed onto the wind index. In the Tropics we see the strengthening of the Hadley cell associated with El Niño. The first part of this mechanism is confirmed in that the regions of extratropical cooling are collocated with ascent. (The correspondence during SON in the Northern Hemisphere is poor but this relationship holds up in the other seasons not shown.) In Fig. 11 we show the computed vertical pressure velocity also regressed onto the wind index. The regions of extratropical ascent that force the cooling are largely captured by the relationship described by Eq. (7), supporting the theory that the extratropical vertical velocity is eddy induced.

The climatological forcing of $\langle\bar{v}\rangle$, and hence $\langle\bar{\omega}\rangle$, by the momentum flux convergence of transient eddies, $-\partial\left(\left\langle\overline{u^{\prime} v^{\prime}}\right\rangle \cos ^{2} \phi\right) / a \partial \phi$, gives cooling in the Tropics and high latitudes and warming in the midlatitudes, centered around $30^{\circ}-40^{\circ} \mathrm{N}$ and $\mathrm{S}$. The pattern of the anomalous $\left.-\partial\left(\overline{\left\langle u^{\prime} v^{\prime}\right.}\right\rangle \cos ^{2} \phi\right) / a \partial \phi$, shown in Fig. $6 c$ has a variable relationship to the climatological pattern. In the Southern Hemisphere the anomalous eddy-induced vertical velocity primarily weakens the circulation. In the Northern Hemisphere an equatorward shift of the pattern of eddy-induced vertical motion is also important: midlatitude cooling is partially explained by a southward movement of the latitude of eddy-induced subsidence to be replaced by weaker subsidence or even ascent.

With the extratropical temperature anomalies shown to be forced by eddy-induced vertical motions we are left to justify only the link between the tropically forced 
(a) JJA

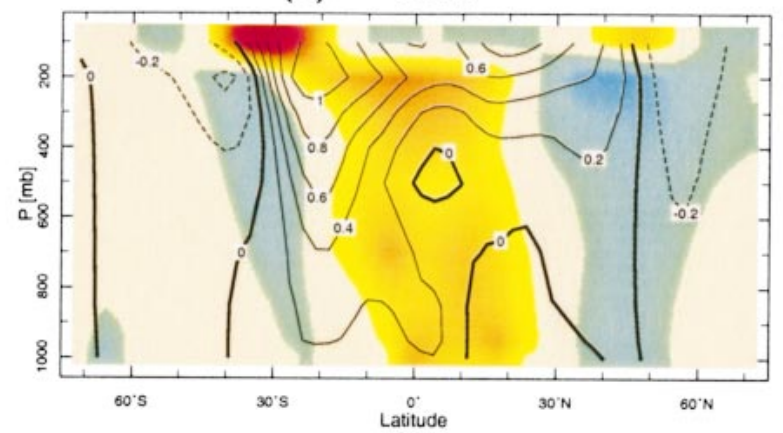

(b) SON

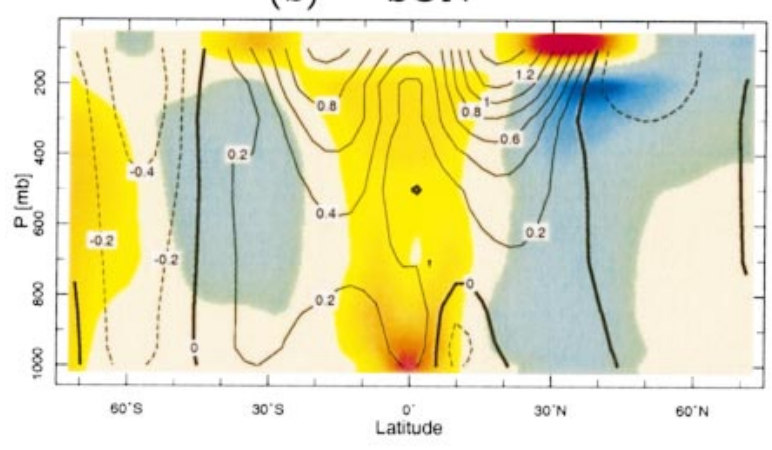

(c) DJF

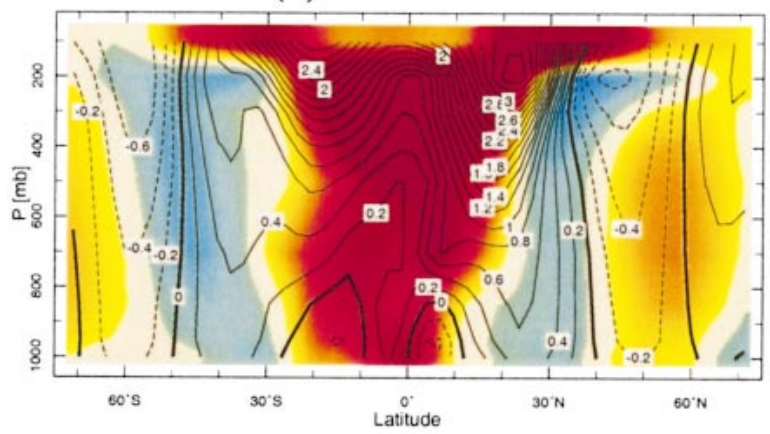

(d) MAM

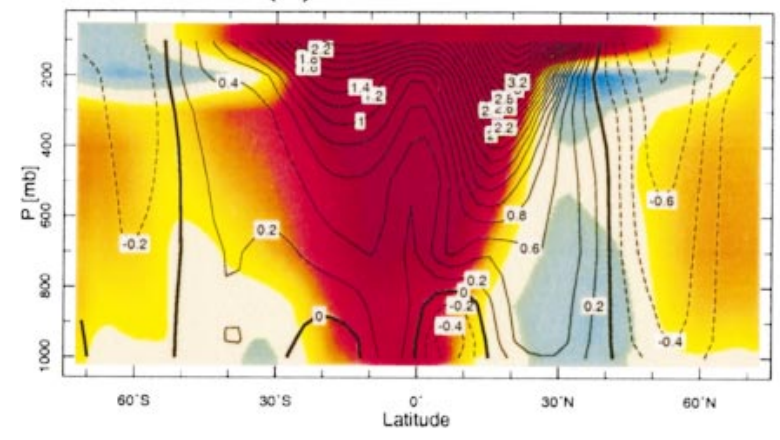

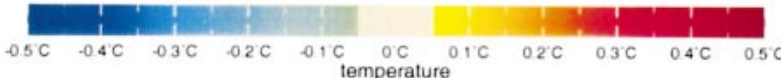

changes in the strength and latitude of the subtropical jets and the changes in the meridional distribution of transient eddy momentum flux convergence.

\section{El Niño-induced changes in eddy fluxes and temperature in a linear model}

In this section we propose a mechanism by which the anomalous zonal wind acceleration can induce eddy momentum flux anomalies that cause the observed midlatitude cooling during El Niño. The anomaly in the subtropical jet entails an increase in vertical wind shear. By thermal wind balance, this is associated with an increase in baroclinicity and increased heat fluxes. However, the jet anomaly will also affect the propagation of transient waves. Since wave propagation is directly related to the eddy fluxes, with a poleward momentum flux indicating equatorward wave propagation, and a poleward heat flux indicating upward wave propagation (Edmon et al. 1980), a change in wave propagation entails a change in eddy fluxes. We will show that the change in wave geometry is such that it primarily affects wave propagation in the meridional direction. Since this process is not as strongly tied to the anomalous temperature field as the change in eddy heat flux is, its effect on temperature can be to enhance, rather than reduce, the initial temperature gradient anomaly.

We use a model to diagnose the basic-state wave propagation characteristics, first for the climatological DJF basic state, and then for an El Niño state that has an added westerly anomaly in the subtropics. We then examine the changes in wave structure and fluxes that correspond to these changes in basic state, and deduce the related eddy-driven temperature anomaly in midlatitudes.

\section{a. The diagnostic approach}

We use a linear quasigeostrophic (QG) model, in which we force waves at the surface. This calculation, which is standard for stationary waves (e.g., Matsuno 1970), is applied here to transient waves that are baroclinically unstable. Our approach assumes that wave propagation in the vertical and meridional plane is relevant for understanding the structure of baroclinically unstable waves. This is based on the overreflection view of baroclinic instability (e.g., Lindzen et al. 1980). The validity of the underlying assumption - that WentzelKramers-Brillouin (WKB) holds at least in most of the domain-is discussed in some detail in the context of baroclinic instability in Lindzen and Rosenthal (1981;

FIG. 9. The zonal-mean zonal wind (contours, $\mathrm{m} \mathrm{s}^{-1}$ ) and zonal mean temperature (colors, K) from the ECHAM-4.5 atmospheric GCM forced by observed SSTs, both regressed onto the PC of the first EOF of an analysis of the model zonal mean zonal wind at $300 \mathrm{mb}$. Results are for (a) JJA, (b) SON, (c) DJF, and (d) MAM. 
(a) SON

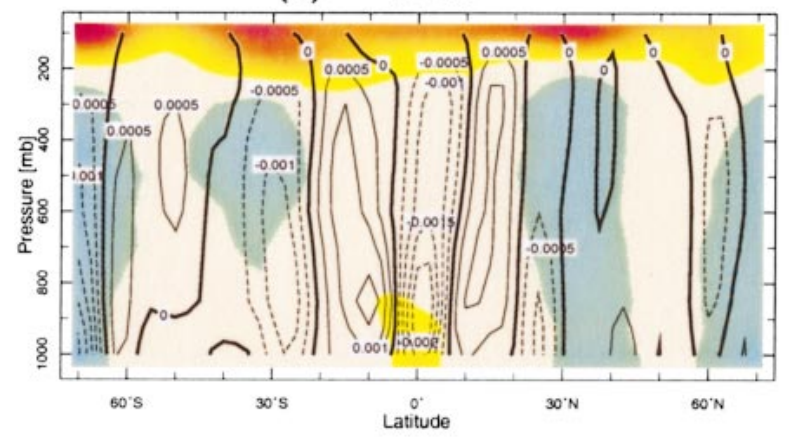

(b) DJF

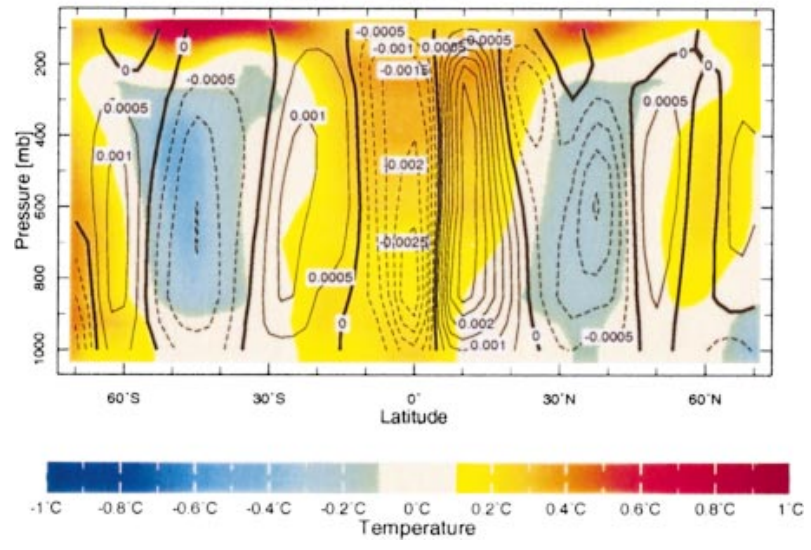

FIG. 10. The zonal-mean vertical pressure velocity (contours, $\mathrm{Pa}$ $\mathrm{s}^{-1}$ ) and zonal-mean temperature (colors, $\mathrm{K}$ ) both regressed onto the time series of Fig. $1 \mathrm{~b}$ for (a) SON and (b) DJF.

see also Lindzen et al. 1980), and for stratospheric stationary waves in Harnik and Lindzen (2001).

Our calculation is an extension of Harnik and Lindzen (2001, hereafter referred to as HL) to transient waves. For brevity, we only review the basic wave propagation theory relevant to our calculation here, and discuss the applicability of our approach and its limitations in the appendix.

The quasigeostrophic equation of conservation of potential vorticity (PV), linearized about a zonal-mean basic state, yields a wave equation, which can be written for the geopotential height perturbation (e.g., Matsuno 1970). Using spherical coordinates, and assuming a sinusoidal structure in longitude, with a given zonal wavenumber $s$, and frequency $\sigma$, we get

$$
\begin{aligned}
& \frac{a^{2} f^{2}}{N^{2}} \frac{\partial^{2} \varphi}{\partial z^{2}}+\frac{f}{\cos \phi} \frac{\partial}{\partial \phi}\left[\cos \phi \frac{\partial}{\partial \phi}\left(\frac{\varphi}{f}\right)\right] \\
& +\left[\frac{a\left\langle\overline{q_{\phi}}\right\rangle}{\langle\bar{u}\rangle-\frac{\sigma a \cos \phi}{s}}-\frac{s^{2}}{\cos ^{2} \phi}+a^{2} f^{2} F\left(N^{2}\right)\right] \varphi \\
& \quad=\text { damping. }
\end{aligned}
$$

A full derivation is presented in HL. Here, $z$ is the log pressure vertical coordinate, $\langle\bar{u}\rangle$ is the zonal mean wind, $\left\langle\overline{q_{\phi}}\right\rangle$ is the meridional gradient of zonal mean potential vorticity, $N$ is the Brunt-Väisälä frequency, $\rho$ is density, and $F\left(N^{2}\right)$ is a function of $N^{2}$ (see HL). The variable $\varphi$ is the wave geopotential streamfunction, which is related to the actual wave geopotential height field $\Phi$ as follows

$$
\Phi=\varphi e^{i(s \lambda-\sigma t)} \sqrt{\frac{N}{\rho}} .
$$

Here, $\lambda$ and $t$ are longitude and time coordinates, respectively. The frequency $\sigma$ can be a complex number, with the real part $\left(\sigma_{r}\right)$ yielding the phase speed, and the imaginary part $\left(\sigma_{i}\right)$ being an exponential growth rate. Including a growth rate is important because it results in a decrease of wave amplitude and fluxes as the waves propagate upward and equatorward from their source in the lower troposphere, much like damping would [for the physical relation between growth and damping see Charney and Pedlosky (1963)].

The first two terms on the left-hand side of Eq. (8) represent vertical and meridional propagation, respectively. The second term in brackets on the left-hand side of Eq. (8) is closely related to the square of the index of refraction (Matsuno 1970): ${ }^{4}$

$$
n_{\mathrm{ref}}^{2}=\frac{a\left\langle\overline{q_{\phi}}\right\rangle}{\langle\bar{u}\rangle-\frac{\sigma_{r} a \cos \phi}{s}}-\frac{s^{2}}{\cos ^{2} \phi}+a^{2} f^{2} F\left(N^{2}\right) .
$$

Equation 8 tells us how a wave of given zonal wavenumber and frequency propagates in the vertical and meridional plane, with the important basic-state quantity for wave propagation being the index of refraction. The solution has a sinusoidal form (wave propagation) in regions where $n_{\text {ref }}^{2}>0$ and exponential form (wave evanescence) in regions where $n_{\text {ref }}^{2}<0$. Karoly and Hoskins (1982) showed that in regions of wave propagation, wave packets tend to refract toward large values of the index of refraction. According to Eq. 8, the basic state affects the index of refraction primarily through the meridional gradient of PV. The zonal wind affects $n_{\text {ref }}^{2}$ most where $\langle\bar{u}\rangle=\sigma_{r} a \cos \phi / s$ and $n_{\text {ref }}^{2}$ becomes infinite, which is referred to as the critical surface. Linear waves reaching critical surfaces will be absorbed, in the presence of even the tiniest amount of damping (Dickinson 1968).

To relate the variations in the index of refraction to changes in wave structure we separate the effects of the index of refraction on vertical and meridional propagation. The meridional and vertical parts of the index of refraction are the meridional $(l)$ and vertical

\footnotetext{
${ }^{4}$ Defining $n_{\text {ref }}^{2}$ using the real part of the frequency allows us to distinguish between spatial decay that is due to the growth rate, and decay that is due to the basic state not supporting wave propagation (see following text).
} 
(a) SON

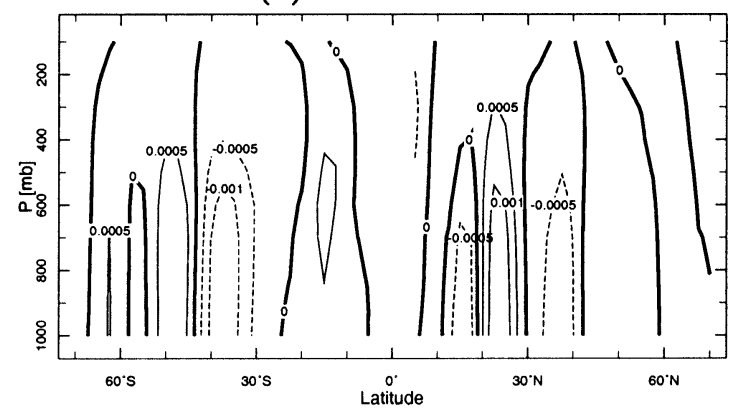

(b) DJF

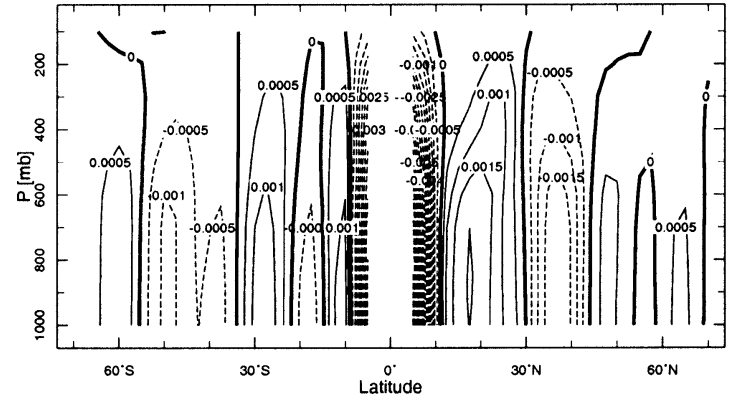

FIG. 11. The eddy-induced zonal-mean vertical pressure velocity $\left(\mathrm{Pa} \mathrm{s}^{-1}\right.$ ) computed from Eq. (6) for (a) SON and (b) DJF regressed onto the wind index.

(m) wavenumbers (see HL). Analogous to the effects of $n_{\text {ref }}^{2}$, waves propagate (are evanescent) in the meridional direction in regions where $l^{2}>0\left(l^{2}<0\right)$, and surfaces of $l^{2}=0$ reflect the waves. Furthermore, waves tend to meridionally propagate toward high values of the meridional wavenumber, and away from low values.

\section{b. The model setup}

The model, which is centered around Eq. (8), is on a sphere, and extends from the surface to $49 \mathrm{~km}$. We have a sponge layer above about $40 \mathrm{~km}$, and equatorwards of about $20^{\circ}$ latitude, to represent thermal and momentum damping by gravity waves in the upper stratosphere ${ }^{5}$ and absorption at the tropical critical surface, or radiation to the other hemisphere (see HL for more details). We specify a zonal wavenumber of $s=$ 6 , a frequency of $\sigma_{r}=1.3 \mathrm{day}^{-1}$ (which corresponds to a zonal phase speed of $13 \mathrm{~m} \mathrm{~s}^{-1}$ at $35^{\circ}$ latitude), and an exponential growth rate of $\sigma_{i}=0.25$ day $^{-1}$, which are within the observed range for midlatitude eddies [see Figs. 3 and 4 of Chang (1999)]. We force the model by specifying the amplitude of $\varphi / f$ at the

\footnotetext{
${ }^{5}$ Since we are looking at medium-scale waves that do not propagate in the stratosphere (Charney and Drazin 1961), the form of the top sponge layer does not really matter.
}

surface to be constant with latitude, ${ }^{6}$ and, given the basic state, solve Eq. (8) for the geopotential structure as a function of latitude and height. Once the geopotential structure is known the vertical and meridional wavenumbers can be deduced as described above (and detailed in HL) and the eddy momentum and heat fluxes can be computed using the QG assumptions. This allows us to see the effect that a change in the zonal wind structure has on the wave geometry and wave fluxes.

\section{c. Climatology}

Figure 12 shows the climatological DJF zonal mean wind (thin contours), which we use for our climatology model run. The corresponding meridional gradients of PV (not shown) have a maximum along the jet axis, reaching maximum values slightly below the jet peaks.

Figure 13a shows the corresponding meridional wavenumber for the Northern Hemisphere climatology run. There are two ridges that act as waveguides, one aligned with the jet and another in midlatitudes. To illustrate the effect of the meridional waveguide on wave propagation we use ray tracing (Karoly and Hoskins 1982). This shows the path that a wave packet will follow when emanating from a point source at a given initial propagation angle. In Fig. 13a we show wave rays emanating from two point sources (the circled stars), one in each of the two waveguides. For each point we choose two initial propagation directions, and calculate the corresponding wave rays for the climatology (solid lines for the climatology). We see that there is some leakage between the two waveguides, such that for some initial angles, rays starting in one waveguide will end up in the other. The downward refraction of the rays is a result of there being a reflective surface for vertical propagation in the upper troposphere, as expected for synoptic-scale waves (Charney and Drazin 1961).

To examine the effect that these changes in wave geometry have on wave propagation, it is helpful to think in terms of the Eliassen-Palm (EP) fluxes (Edmon et al. 1980). The vertical component of the EP flux is proportional to the eddy heat flux, while the meridional component is proportional to the negative of the eddy momentum flux. The EP fluxes for the climatology run (obtained from our model wave solution) are upward and equatorward, consistent with the observed (Edmon et al. 1980).

\footnotetext{
${ }^{6}$ Here, $\varphi / f$ is the geopotential streamfunction in the version of QG on spherical coordinates that we are using (see HL). Based on various runs we have done with tropospheric waves, and various shapes of forcing at the surface, we find that while the shape of the forcing does affect the wavenumbers in a large part of the troposphere, the main features are robust, and the best results, in terms of wave propagation characteristics, are obtained when we use a geopotential height amplitude that is constant in latitude.
} 


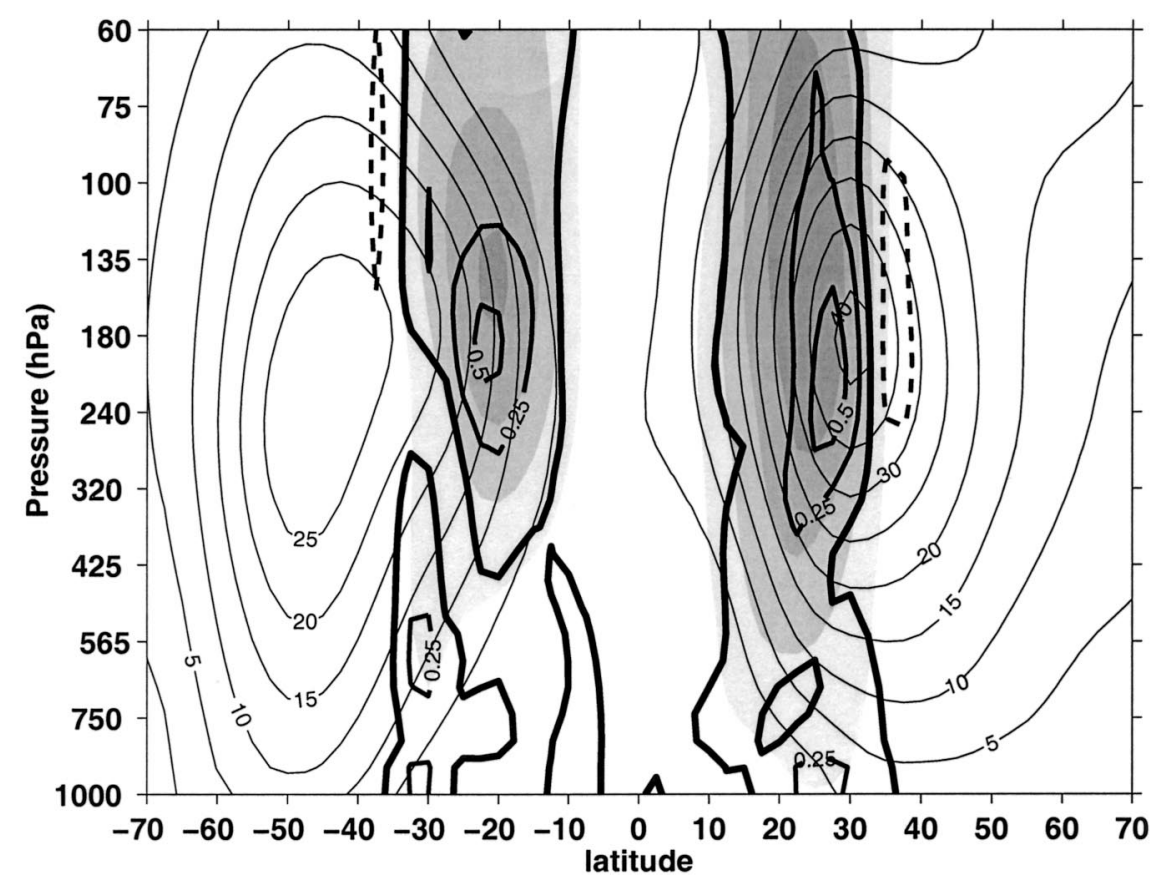

FIG. 12. DJF climatological zonal-mean wind (thin contours, intervals of $5 \mathrm{~m} \mathrm{~s}^{-1}$ ), the El Niño wind anomalies used in our calculation (shaded, values between 0.5 and $3 \mathrm{~m} \mathrm{~s}^{-1}$, at intervals of 0.5 ), and the corresponding meridional PV gradient anomalies (thick lines, zero line thickest, negative values dashed, contour interval of $\left.0.25 \times 10^{-11} \mathrm{~m}^{-1} \mathrm{~s}^{-1}\right)$.

\section{d. The El Niño-related anomalies}

Figure 12 shows the zonal wind anomaly (shaded) that is added to the climatology, to represent the "El Niño" run. Note that we cannot just impose the observed change in the jet because this already includes the effect of the eddies. We therefore assume that the midlatitude eddies mostly affect the zonal mean wind in midlatitudes, and consequently impose the observed wind anomalies only in the subtropics (the sensitivity to the choice of perturbation is discussed in the appendix). The Southern Hemisphere anomalous wind pattern (Fig. 4c) seems to support our assumption-the subtropical upper-tropospheric positive anomaly is El Niño driven, while the anomalous dipole centered at $45^{\circ} \mathrm{S}$ is eddy-driven. ${ }^{7}$ We use the observed positive wind anomalies between $30^{\circ} \mathrm{S}$ and $40^{\circ} \mathrm{N}$ (from Fig. 4c), set the anomalies to zero elsewhere, and apply some smoothing at the interface. Also shown are the corresponding changes in meridional PV gradients. We see that the PV gradients increase in the region of the wind perturbation, and decrease poleward and equatorward of it.

Figure $13 \mathrm{~b}$ shows the corresponding changes in $n_{\mathrm{ref}}^{2}$ (El Niño minus climatology) for the Northern Hemisphere, along with the critical surface for both basic

\footnotetext{
${ }^{7}$ This is consistent with our expectation that eddy-driven anomalies will extend to the surface, while the anomalies driven by tropical heating will be largest in the upper troposphere (Lee and Kim 2003).
}

states. The critical surface shifts slightly equatorward, resulting in very large and negative anomalies of $n_{\text {ref }}^{2}$ surrounding it (white regions surrounding the critical surfaces). The value $n_{\text {ref }}^{2}$ decreases in most places because $\langle\bar{u}\rangle$ increases. The decrease in $n_{\text {ref }}^{2}$ is smallest where $\left\langle\overline{q_{\phi}}\right\rangle$ increases along with $\langle\bar{u}\rangle$ (between $25^{\circ}$ and $35^{\circ} \mathrm{N}$ ) and is largest where $\left\langle\overline{q_{\phi}}\right\rangle$ gets smaller (between $33^{\circ}$ and $38^{\circ} \mathrm{N}$ ).

Most of the variation in $n_{\mathrm{ref}}^{2}$ is accounted for by a change in the meridional wavenumber, which is shown in Fig. 14a, along with the Southern Hemisphere anomalies (which were calculated separately then plotted on one graph). There are vertical regions of large negative anomalies in meridional wavenumber at around $35^{\circ}$ latitude in both hemispheres that correspond to local minima in the $n_{\text {ref }}^{2}$ anomaly. The region of large and negative meridional wavenumber anomalies in the El Niño run (Fig. 14a) lies in the climatological meridional wavenumber trough that separates the two waveguides (Fig. 13a). The El Niño wind anomaly essentially causes the two waveguides to be more separated than for climatology.

The El Niño minus climatology EP fluxes are plotted on top of the meridional wavenumber anomalies in Fig. 14a (arrows). The anomalous fluxes are upward (indicating larger meridional heat fluxes) in the El Niño run, consistent with the increase in shear (and the observations). More important, we see that the anomalous 
(a) meridional wave\# (shaded), wave rays (climatology -, El Nino --)

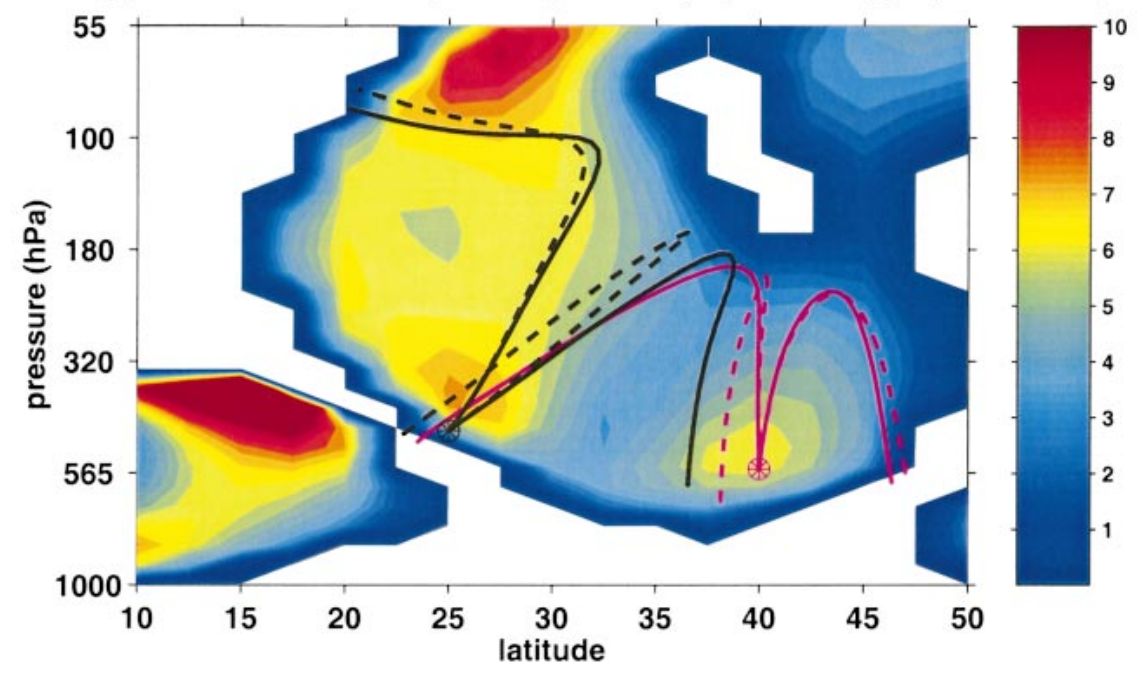

(b) $\mathrm{n}_{\text {ref }}^{2}$ (shaded), critical surface (climatology -, El Nino --)

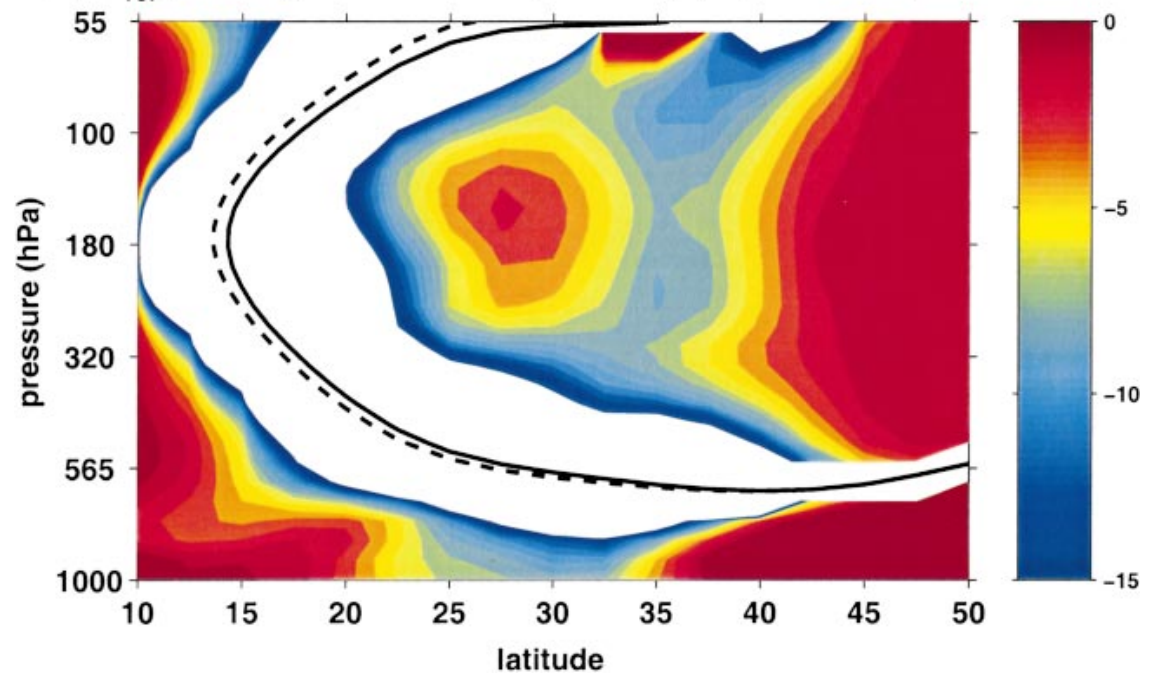

FIG. 13. (a) The climatological meridional wavenumber (units of $\mathrm{yr}^{-1}$, color) and wave packet rays, for the climatology (solid black and magenta lines) and El Niño (dashed). Circled stars show the source for the ray tracing calculations. (b) The $n_{\text {ref }}^{2}$ anomalies (El Niño minus climatology, color) and the critical surface (where $\langle\bar{u}\rangle$ equals the phase speed of the wave, black lines) for the climatology (solid) and El Niño (dashed).

fluxes diverge away from the vertical regions of negative wavenumber anomalies, making the EP flux more equatorward on its equatorward side and more poleward on its poleward side. Since the climatological meridional EP fluxes are equatorward everywhere this corresponds to an intensification of the fluxes on the equatorward side and a weakening on the poleward side.

The argument that the differences in EP fluxes are due to the deeper separation between the two waveguides in the El Niño case is clearly illustrated by the ray tracing calculations (lines in Fig. 13a). We see the leakage between the two waveguides for both the cli- matology (solid) and the El Niño, but we find that the angle needed for rays to cross from one waveguide to the other is different, such that a smaller portion of the rays cross from one waveguide to the other in the El Niño case, consistent with a larger separation between the two waveguides, and with the EP flux anomaly pattern of Fig. 14a.

The anomalous momentum flux pattern in our model is a dipole, with a positive peak at around $25^{\circ} \mathrm{N}$ and a negative peak around $43^{\circ} \mathrm{N}$. This is similar to the observed momentum flux anomaly during El Niño (the convergence of which is shown in Fig. 6c), but is shifted 

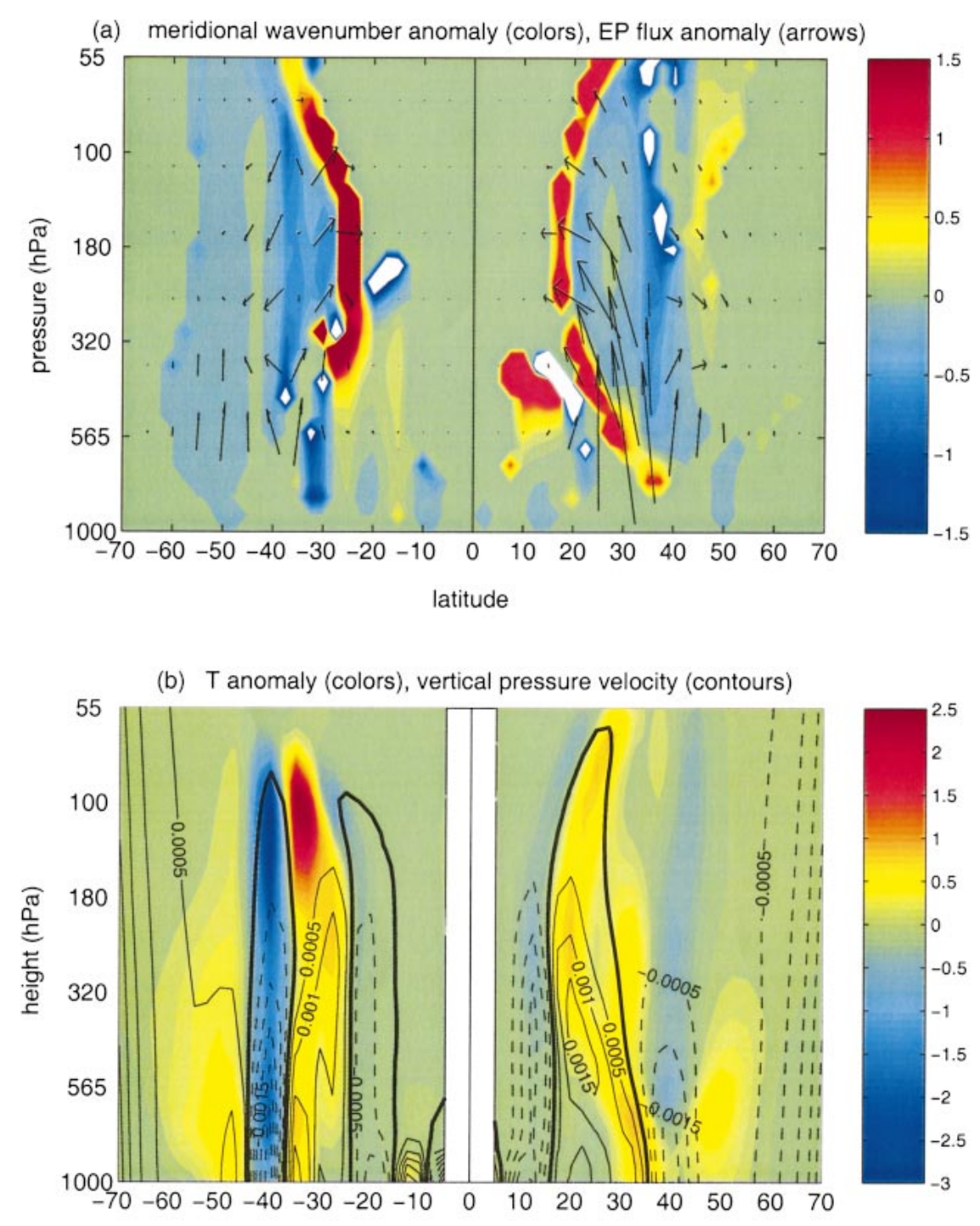

latitude

FIG. 14. (a) The anomaly in meridional wavenumber (El Niño minus the climatology, units of $\mathrm{yr}^{-1}$, colors), and the anomalous EP fluxes (arrows). (b) The anomalous mean vertical pressure velocity ( $\mathrm{Pa} \mathrm{s}^{-1}$, black contours, negative values dashed, zero line thick) and the corresponding temperature anomalies $\left({ }^{\circ} \mathrm{C}\right.$, colors, see text for details).

poleward (the observed positive and negative in momentum fluxes are around $20^{\circ}$ and $35^{\circ} \mathrm{N}$ ).

\section{e. The implications for temperature}

The implied change in temperature $(\Delta\langle\bar{T}\rangle)$ can be derived from Eq. (6) (neglecting the $\omega^{\prime}$ terms), with $\langle\bar{\omega}\rangle$ given by Eq. (7), using the changes in the transient momentum and heat fluxes computed by the QG model using its Newtonian damping distribution. Figure 14b shows $\Delta\langle\bar{\omega}\rangle$, along with the corresponding $\Delta\langle\bar{T}\rangle$. We see regions of upward velocity, with corresponding cooling, extending in height over much of the troposphere, on the poleward sides of the anomalous EP flux divergence, with adjacent regions of downward velocity, and warming on the equatorward side. The temperature anomalies are smaller in the Northern Hemisphere than in the Southern Hemisphere, but the actual values are arbitrary, depending on the Newtonian damping used, and on the assumption of no momentum damping. The results are qualitatively similar to the observed temperature distribution, but the regions of cooling are narrower, and more poleward than observed. More detailed agreement would not be expected: the model only rep- 
resents the initial response of the eddies to the changes in the subtropical jet, assumes only one zonal wavenumber, and is highly simplified. Previous work has suggested that the eddy momentum fluxes may be sensitive to the basic-state wind during later stages of the baroclinic life cycle (Simmons and Hoskins 1980; see also Thorncroft et al. 1993; Balasubramania and Garner 1997). Our use of the observed wind anomaly in the subtropics, which already includes some of the effect of the eddies, may also affect our results.

Because of the limitations of the approach these results are preliminary but they nonetheless suggest that changes in transient eddy momentum fluxes may be caused by wave propagation patterns responding to El Niño-induced changes in the meridional structure of the jet. Moreover, these changes are capable of inducing a midlatitude cooling as observed, in spite of the anomalous increase in eddy heat fluxes that tends to warm midlatitudes.

\section{Discussion}

The mechanism proposed here for how the Hadley cell influences higher-latitude climate variability is similar to that advanced by Chang (1995) and Chang (1998) who appealed to an anomalous eddy-driven mean meridional circulation. Chang suggests that the temperature anomalies are caused by latitude shifts of the descending branch of the Hadley cell and the eddy-driven mean meridional circulation. However, the anomalies studied here can arise from either a latitude shift, or a change in strength, of the eddy-driven mean meridional circulation. Both are explained by the zonal-mean zonal wind anomaly creating a region of anomalously low meridional wavenumber in the mid and upper troposphere of the subtropics that diverts transient eddies to the north and south. The exact latitude of this region of anomalously low meridional wavenumber is determined by the latitude of the change in zonal wind. The results of the QG model show that, depending on the latitude of the wind anomaly, the resulting eddy momentum flux anomalies can either change the strength of, or latitudinally shift, the eddy-driven mean meridional circulation. What is important and robust is not whether the anomalies appear as a shift or a weakening of the pattern but that, at some latitude poleward of the stronger subtropical jet, eddy momentum fluxes induce cooling.

Our proposed mechanism is distinctly different to that proposed by Hou (1998; see also Hou 1993; Hou and Molod 1995). He argues that changes in the Hadley cell intensity cause a change in baroclinicity in the subtropics that results in a change in the transient eddy heat flux that then drives the extratropical temperature changes. It is clearly shown in the observational analysis presented here that, for ENSO-induced changes, the transient eddy heat fluxes act to damp the temperature anomalies forced by the mean meridional circulation and do not create them. This reality was reproduced in the linear QG model results and in the GCM results.

\section{Conclusions}

We have examined the mechanisms whereby climate varies in a way that is symmetric about the equator. In the recent period of high-quality observations hemispherically symmetric variability is associated with ENSO. During an El Niño event the tropical atmosphere warms but the midlatitudes cool. The midlatitude cooling occurs in all seasons and at all longitudes (except for northern North America during local winter). This pattern of climate variability has a large zonally symmetric component and is also symmetric about the equator. We have sought an explanation for why there are zonal strips in the midlatitudes of each hemisphere where the temperature change is opposite to that in the Tropics.

Our findings are as follows, described for the case of an El Niño event (the opposite sequence occurs for a La Niña event).

- The tropical atmosphere warms, presumably due to heat release from the tropical Pacific Ocean. The warming is spatially uniform between about $20^{\circ} \mathrm{N}$ and $20^{\circ} \mathrm{S}$ because the tropical atmosphere cannot maintain horizontal temperature gradients (Schneider 1977; Held and Hou 1980). By thermal wind balance the subtropical jets in both hemispheres strengthen on the poleward edge of the tropical warming. Since the warming occurs at all longitudes so does strengthening of the jets.

- A strengthened subtropical jet, and associated changes in the meridional gradient of potential vorticity, strengthens the midlatitude region where transient eddies tend to adopt a low meridional wavenumber, resulting in eddies being refracted away from this area, creating the observed patterns of eddy momentum fluxes.

- Changes in the transient eddy zonal momentum flux convergence apply a westerly force between $30^{\circ}$ and $45^{\circ}$ in both hemispheres and an easterly force between $45^{\circ}$ and $60^{\circ}$. This is balanced by the Coriolis torque creating equatorward upper-tropospheric meridional winds equatorward of about $45^{\circ}$ and poleward winds poleward of $45^{\circ}$.

- The eddy-induced mean meridional circulation creates ascent in midlatitudes. The ascent causes adiabatic cooling, which is balanced by diabatic warming (presumably reduced radiative cooling).

- The transient eddy heat flux opposes these changes by cooling the Tropics and warming the midlatitudes but cannot overwhelm the effects of the mean meridional circulation.

- The result is that during El Niño events large areas of the midlatitudes cool. The cooling is quite modest, rarely reaching $1^{\circ} \mathrm{C}$ for a typical occurence, but occurs 
at all longitudes and from the ocean surface to the upper troposphere.

The mechanism whereby changes in the subtropical jets impact the transient eddies and the eddy-driven mean meridional circulation is a way whereby tropical variability influences extratropical climate that is fundamentally different from the more familiar teleconnections route. Teleconnections, which work by Rossby wave propagation (e.g., Hoskins and Karoly 1981), create zonally asymmetric climate anomalies and tend to work primarily during local winter. In contrast the mechanism described here works simultaneously in both hemispheres and at all longitudes causing climate anomalies that are hemispherically and zonally symmetric. It also allows the Tropics to influence extratropical climate outside of the winter season. It is anticipated that the same physics of eddy-mean flow interactions could be involved in the atmospheric response to other forcings, for example, orbitally forced variations in ENSO and the strength of the Hadley cell (Clement et al. 1999, 2001; Lindzen and Pan 1994), changes in the Hadley cell forced by rising greenhouse gases (Dai et al. 2001), or other mechanisms that directly impact the tropospheric jet. The mechanisms described here provide one way in which paleoclimatic evidence of climate changes that were the same irrespective of longitude and latitude can be explained, raising the question of what caused the required change in the coupled climate of the Tropics. Stott et al. (2002) and Koutavos et al. (2002) have recently presented evidence that cold conditions in the extratropics (e.g., the Last Glacial Maximum) were associated with El Niño-like conditions. The mechanisms described here may help provide the reason why.

Acknowledgments. This work was supported by NOAA Grant UCSIO-CU-02165401SCF, NOAA Grant NA16GP2024, and NSF Grants ATM-9986515 and ATM-9986072. We would like to thank Adam Sobel, Huei-Ping Huang, and Mark Cane for useful conversations. We thank David DeWitt and Lisa Goddard and all at the International Research Institute for Climate Prediction for excellent and swift cooperation. The comments and criticisms of three anonymous reviewers led to an improved manuscript.

\section{APPENDIX}

\section{Caveats Regarding Use of the Linear Steady-State QG Model for Determining the Anomalous Eddy- Driven Cooling Patterns}

In this appendix we discuss the limitations of the model calculation of section 5 to explain the observed eddy-induced heating and cooling patterns.

Our calculation is based on the assumption that the subtropical wind anomaly affects the waves mostly through changes in the basic-state index of refraction. In fact waves are also strongly affected by the global distribution of sources and sinks. Essentially, waves reach an equilibrium between their sources (baroclinic instability for transient, midlatitude tropospheric waves) and their sinks. The main sinks of transient tropospheric wave activity are thermal damping and nonlinear dissipation through wave breaking near the critical surface. In our model we use Newtonian damping on temperature, and a constant damping on potential vorticity (with a damping rate of $\sigma_{i}$ ). The nonlinear damping of the waves in the upper troposphere is crudely parameterized, since waves are absorbed at a critical surface in the presence of even the smallest amount of damping. If the perturbation in the mean flow mostly affects the index of refraction, and not the thermal and momentum wave damping timescales, the relation between anomalies in wave propagation and the index of refraction will be close.

The sensitivity of our results to the form of wind perturbation and basic state can be judged by comparing the two hemispheres (Figs. 11 and 13). As in the Northern Hemisphere, the meridional wavenumber anomalies in the Southern Hemisphere also have a vertical region of large negative values $\left(30^{\circ}-40^{\circ} \mathrm{S}\right)$, which coincides with a trough between two waveguides (not shown). The Southern Hemisphere basic-state geometry, however, is slightly more complex, since the critical surface intersects the "low-latitude waveguide," making it much more narrow in latitude. As a result, the negative and positive wavenumber anomaly regions are much closer to each other, compared to the Northern Hemisphere. In addition, there is a critical surface on the poleward side of the Southern Hemisphere jet, which absorbs any waves that reach it. This can explain why the poleward component of the anomalous EP fluxes is larger in the Southern Hemisphere.

We also tested the sensitivity of our results to the form of wind anomaly, by repeating our calculations for the Northern Hemisphere with the same wind anomaly, shifted poleward. We found that the meridional wavenumbers generally decrease where the meridional PV gradients decrease, and vice versa, but the magnitude is sensitive to the basic-state structure, so that changes are largest at the edges of the waveguides, and in the troughs in between, and the anomalous momentum fluxes split at the latitude of the strongest decrease in meridional wavenumber. A wind anomaly that is localized enough in latitude will have regions of negative PV gradient anomalies at its sides. In most cases, one of these negative regions will lie at one of the waveguide edges, resulting in a decrease in meridional wavenumber, and a corresponding latitudinally localized region of anomalous momentum flux divergence. This, in turn, will result in a region of anomalous cooling. The qualitative nature of our results, therefore, is robust, even though the details in terms of wave geometry depend on the specific basic state and wind anomalies.

Finally, a note is needed about the use of our steadystate wave solution to calculate the corresponding wave 
fluxes. In principle, we should solve an eigenvalue problem for a given zonal wavenumber to get the phase speed and growth rate of the wave and its corresponding structure. The eigenvalue solution is the one that can be maintained without forcing. This means, for example, that if we initialize a time-dependent model with this solution, the structure, phase speed, and growth rate of the wave will not change with time. On the other hand, if we initialize a time-dependent model with one of our steady-state wave solutions, the phase speed and wave structure will adjust toward the eigenvalue solution for the specified zonal wavenumber. We can get an idea of how large these structure changes are by varying the phase speed and growth rate in our model. We find that this does not affect our results qualitatively.

\section{REFERENCES}

Arkin, A., 1982: The relationship between interannual variability in the $200 \mathrm{mb}$ tropical wind field and the Southern Oscillation. Mon. Wea. Rev., 110, 1393-1404.

Balasubramania, G., and S. T. Garner, 1997: The role of momentum fluxes in shaping the life cycle of a baroclinic wave. J. Atmos. Sci., 54, 510-533.

Bush, A. B. G., and S. G. H. Philander, 1998: The role of oceanatmosphere interactions in tropical cooling during the last glacial maximum. Science, 279, 1341-1344.

Chang, E. K. M., 1995: The influence of Hadley circulation intensity changes on extratropical climate in an idealized model. J. Atmos. Sci., 52, 2006-2024.

_ 1998: Poleward-propagating angular momentum perturbations induced by zonally symmetric heat sources in the Tropics. $J$. Atmos. Sci., 55, 2229-2248.

_ 1999: Characteristics of wave packets in the upper troposphere. Part II: Hemispheric and seasonal differences. J. Atmos. Sci., 56, 1729-1747.

Charney, J. G., and P. G. Drazin, 1961: Propagation of planetary scale disturbances from the lower into the upper atmosphere. J. Geophys. Res., 66, 83-110.

— waves in the atmosphere. J. Geophys. Res., 68, 6441-6442.

Clement, A. C., R. Seager, and M. A. Cane, 1999: Orbital controls on the El Niño/Southern Oscillation and the tropical climate. Paleoceanography, 14, 441-456.

- M. A. Cane, and R. Seager, 2001: An orbitally driven tropical source for abrupt climate change. J. Climate, 14, 2369-2375.

Dai, A., T. Wigley, B. Boville, J. Kiehl, and L. Buja, 2001: Climates of the twentieth and twenty-first centuries simulated by the NCAR Climate System Model. J. Climate, 14, 485-519.

Denton, G. H., and C. H. Hendy, 1994: Younger Dryas age advance of Franz-Josef glacier in the Southern Alps of New Zealand. Science, 264, 1434-1437.

Dickey, J. O., S. L. Marcus, and R. T. Hide, 1992: Global propagation of interannual fluctuations in atmospheric angular momentum. Nature, 357, 484-488.

Dickinson, R. E., 1968: Planetary waves propagating vertically through weak westerly wind wave guides. J. Atmos. Sci., 25, 984-1002.

Edmon, H., B. J. Hoskins, and M. E. McIntyre, 1980: Eliassen-Palm cross-sections for the troposphere. J. Atmos. Sci., 37, 2600-2616.

Garreaud, R. D., and D. S. Battisti, 1999: Interannual (ENSO) and interdecadal (ENSO-like) variability in the Southern Hemisphere tropospheric circulation. J. Climate, 12, 2113-2123.

Harnik, N., and R. S. Lindzen, 2001: The effect of reflecting surfaces on the vertical structure and variability of stratospheric planetary waves. J. Atmos. Sci., 58, 2872-2894.
Held, I., and A. Y. Hou, 1980: Nonlinear axially symmetric circulations in a nearly inviscid atmosphere. J. Atmos. Sci., 37, 515533.

Hoerling, M. P., J. S. Whitaker, A. Kumar, and W. Wang, 2001: The midlatitude warming during 1998-2000. Geophys. Res. Lett., 28, $755-758$.

Hoskins, B., and K. Karoly, 1981: The steady response of a spherical atmosphere to thermal and orographic forcing. J. Atmos. Sci., 38, 1179-1196.

Hou, A. Y., 1993: The influence of tropical heating displacements on the extratropical climate. J. Atmos. Sci., 50, 3553-3570.

1998: Hadley circulation as a modulator of the extratropical climate. J. Atmos. Sci., 55, 2437-2457.

- and A. Molod, 1995: Modulation of dynamic heating in the winter extratropics associated with the cross-equatorial Hadley cell. J. Atmos. Sci., 52, 2609-2626.

Kalnay, E., and Coauthors, 1996: The NCEP/NCAR 40-Year Reanalysis Project. Bull. Amer. Meteor. Soc., 77, 437-471.

Karoly, D. J., and B. J. Hoskins, 1982: Three dimensional propagation of planetary waves. J. Meteor. Soc. Japan, 60, 109-123.

Kaser, G., 1999: A review of the modern fluctuations of tropical glaciers. Global Planet. Change, 22, 93-103.

Kistler, R., and Coauthors, 2001: The NCEP-NCAR 50-year reanalysis: Monthly means CD-ROM and documentation. Bull. Amer. Meteor. Soc., 82, 247-268.

Koutavos, A., J. Lynch-Stieglitz, T. Marchitto Jr., and J. P. Sachs, 2002: El Niño-like pattern in Ice Age tropical Pacific sea surface temperature. Science, 297, 226-230.

Lau, N.-C., and M. J. Nath, 1996: The role of the "atmospheric bridge" in linking tropical Pacific ENSO events to extratropical SST anomalies. J. Climate, 9, 2036-2057.

Lee, S., and H.-K. Kim, 2003: The dynamical relationship between subtropical and eddy-driven jets. J. Atmos. Sci., 60, 1490-1503.

Lindzen, R. S., and A. J. Rosenthal, 1981: A WKB asymptotic analysis of baroclinic instability. J. Atmos. Sci., 38, 619-629.

_- and W. Pan, 1994: A note on orbital control of equator-pole heat fluxes. Climate Dyn., 10, 49-57.

_ - B. Farrell, and K. K. Tung, 1980: Concept of wave overreflection and its application to baroclinic instability. J. Atmos. Sci., 37, 44-63.

Lowell, T. V., C. J. Heusser, B. G. Anderson, P. I. Marino, A. Hauser, L. E. Heusser, C. S. D. R. Marchant, and G. H. Denton, 1995: Interhemispheric correlation of late Pleistocene glacial events. Science, 269, 1541-1549.

Markgraf, V., and Coauthors, 2000: Paleoclimate reconstruction along the Pole-Equator-Pole transect of the Americas (PEP 1). Quat. Sci. Rev., 19, 125-140.

Matsuno, T., 1970: Vertical propagation of stationary planetary waves in the winter Northern Hemisphere. J. Atmos. Sci., 27, 871-883.

Robinson, W. A., 2002: On the midlatitude thermal response to tropical warmth. Geophys. Res. Lett., 29, 1190, doi:10.1029/ 2001 GL014158.

Roeckner, E. K., and Coauthors, 1996: The atmospheric general circulation model ECHAM-4: Model description and simulation of present day climate. Max-Planck-Institut für Meteorologie Tech. Rep. 218, 90 pp.

Schneider, E. K., 1977: Axially symmetric steady-state models of the basic state for instability and climate studies. Part II: Non-linear calculations. J. Atmos. Sci., 34, 280-296.

Schneider, N., A. J. Miller, and D. W. Pierce, 2002: Anatomy of North Pacific decadal variability. J. Climate, 15, 586-605.

Seager, R., Y. Kushnir, N. Naik, M. A. Cane, and J. Miller, 2001: Wind-driven shifts in the latitude of the Kuroshio-Oyashio Extension and generation of SST anomalies on decadal timescales. J. Climate, 14, 4249-4265.

Simmons, A. J., and B. J. Hoskins, 1980: Barotropic influences on the growth and decay of nonlinear baroclinic waves. J. Atmos. Sci., 37, 1679-1684.

Stott, L., C. Poulsen, S. Lund, and R. Thunell, 2002: Super ENSO 
and global climate oscillations at millenial timescales. Science, 297, 222-226.

Sun, D.-Z., 2000: The heat sources and sinks of the 1986-87 El Niño. J. Climate, 13, 3533-3550.

Thorncroft, C. D., B. J. Hoskins, and M. E. McIntyre, 1993: Two paradigms of baroclinic-wave life-cycle behaviour. Quart. J. Roy. Meteor. Soc., 119, 17-55.

Wardle, P., 1973: Variations of the glaciers of Westland National Park and the Hooker Range, New Zealand. N. Z. J. Bot., 11, 349388.

Winkler, S., 2000: The "Little Ice Age" maximum in the Southern Alps, New Zealand: Preliminary results at Mueller Glacier. Holocene, 10, 643-647.

Yulaeva, E., and J. M. Wallace, 1994: The signature of ENSO in global temperature and precipitation fields derived from the microwave sounding unit. J. Climate, 7, 1719-1736. 